\title{
Forming Super Star Clusters in the Central Starburst of NGC 253
}

\author{
Leroy, Adam K.
}

2018-12-20

Leroy , A K, Bolatto , A D , Ostriker , E C , Walter , F , Gorski , M , Ginsburg , A , Krieger , N , Levy , R C , Meier, D S, Mills , E, Ott , J , Rosolowsky , E, Thompson, T A, Veilleux , S \& Zschaechner, L K 2018 , ' Forming Super Star Clusters in the Central Starburst of NGC 253 ' , Astrophysical Journal , vol. 869 , no. 2 , 126 . https://doi.org/10.3847/1538-4357/aaecd1

http://hdl.handle.net/10138/298974

https://doi.org/10.3847/1538-4357/aaecd1

unspecified

publishedVersion

Downloaded from Helda, University of Helsinki institutional repository.

This is an electronic reprint of the original article.

This reprint may differ from the original in pagination and typographic detail.

Please cite the original version. 


\title{
Forming Super Star Clusters in the Central Starburst of NGC 253
}

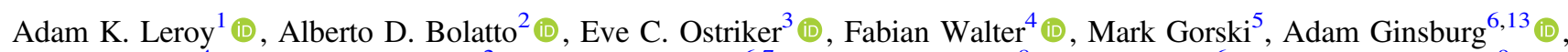 \\ Nico Krieger ${ }^{4}$ (1) Rebecca C. Levy ${ }^{2}$ (1), David S. Meier ${ }^{6,7}$ (1), Elisabeth Mills ${ }^{8}$ (i) , Jürgen Ott ${ }^{6}$ (1) , Erik Rosolowsky $^{9}$ (1) \\ Todd A. Thompson ${ }^{1,10}$, Sylvain Veilleux ${ }^{2}$ (D), and Laura K. Zschaechner ${ }^{11,12}$ (D) \\ ${ }^{1}$ Department of Astronomy, The Ohio State University, 140 West 18th Avenue, Columbus, OH 43210, USA \\ ${ }^{2}$ Department of Astronomy, University of Maryland, College Park, MD 20742, USA \\ ${ }^{3}$ Department of Astrophysical Sciences, Princeton University, Princeton, NJ 08544, USA \\ ${ }^{4}$ Max-Planck-Institut für Astronomie, Königstuhl 17, D-69117, Heidelberg, Germany \\ ${ }^{5}$ Department of Physics and Astronomy, University of Western Ontario, London, Ontario N6A 3K7, Canada \\ ${ }^{6}$ National Radio Astronomy Observatory, PO Box O, 1003 Lopezville Road, Socorro, NM 87801, USA \\ New Mexico Institute of Mining \& Technology, 801 Leroy Place, Socorro, NM 87801, USA \\ ${ }^{8}$ Department of Astronomy, Boston University, 725 Commonwealth Avenue, Boston, MA 02215, USA \\ ${ }^{9}$ Department of Physics, University of Alberta, Edmonton, AB T6G 2E1, Canada \\ ${ }^{10}$ Center for Cosmology \& Astro-Particle Physics, The Ohio State University, Columbus, OH 43210, USA \\ ${ }^{11}$ University of Helsinki, Physicum, Helsingin Yliopisto, Gustaf Hällströmin katu 2, FI-00560 Helsinki, Finland \\ ${ }^{12}$ Finnish Center for Astronomy with ESO, FI-20014 Turun yliopisto, Finland \\ Received 2018 April 5; revised 2018 September 17; accepted 2018 October 20; published 2018 December 18
}

\begin{abstract}
NGC 253 hosts the nearest nuclear starburst. Previous observations show a region rich in molecular gas, with dense clouds associated with recent star formation. We used the Atacama Large Submillimeter/Millimeter Array (ALMA) to image the $350 \mathrm{GHz}$ dust continuum and molecular line emission from this region at $2 \mathrm{pc}$ resolution. Our observations reveal $\sim 14$ bright, compact $(\sim 2-3$ pc FWHM) knots of dust emission. Most of these sources are likely to be forming super star clusters (SSCs) based on their inferred dynamical and gas masses, association with $36 \mathrm{GHz}$ radio continuum emission, and coincidence with line emission tracing dense, excited gas. One source coincides with a known SSC, but the rest remain invisible in Hubble near-infrared (IR) imaging. Our observations imply that gas still constitutes a large fraction of the overall mass in these sources. Their high brightness temperature at $350 \mathrm{GHz}$ also implies a large optical depth near the peak of the IR spectral energy distribution. As a result, these sources may have large IR photospheres, and the IR radiation force likely exceeds $L / c$. Still, their moderate observed velocity dispersions suggest that feedback from radiation, winds, and supernovae are not yet disrupting most sources. This mode of star formation appears to produce a large fraction of stars in the burst. We argue for a scenario in which this phase lasts $\sim 1 \mathrm{Myr}$, after which the clusters shed their natal cocoons but continue to produce ionizing photons. The strong feedback that drives the observed cold gas and X-ray outflows likely occurs after the clusters emerge from this early phase.
\end{abstract}

Key words: galaxies: individual (NGC 253) - galaxies: ISM - galaxies: starburst - galaxies: star clusters: general galaxies: star formation

\section{Introduction}

Vigorous bursts of star formation in galaxy centers and merging galaxies produce super star clusters (SSCs; e.g., Holtzman et al. 1992; Whitmore 2003; Portegies Zwart et al. 2010). The SSCs in well-known local starbursts such as M82 and the Antennae galaxies have been studied for decades (e.g., Whitmore 2003; McCrady et al. 2005). These massive $\left(M_{\star}>10^{5} M_{\odot}\right)$ compact $(R \sim 1 \mathrm{pc})$ concentrations of stars may be younger cousins to the Milky Way's globular clusters.

Less extreme massive young stellar clusters $\left(M_{\star} \gtrsim 10^{4} M_{\odot}\right.$, age $\lesssim 100 \mathrm{Myr}$ ) have been found in the Milky Way and many nearby galaxies (see review by Portegies Zwart et al. 2010; Longmore et al. 2014). Overall, the fraction of stars formed in clusters appears to increase with the surface density of star formation (Kruijssen 2012; Johnson et al. 2016; Ginsburg \& Kruijssen 2018). Because higher levels of star formation activity were prevalent at $z \sim 1-3$, the formation of SSCs may represent a mode of star formation that was more common in the early universe than today. Studying the present-day formation of SSCs may thus offer a window into how star

\footnotetext{
${ }^{13}$ Jansky Fellow.
}

formation proceeded during the epoch of galaxy assembly (e.g., Zhang et al. 2010).

Gas structures associated with recent or future formation of SSCs have been identified in the Antennae galaxies (Herrera et al. 2012; Johnson et al. 2015) and the Large Magellanic Cloud (Ochsendorf et al. 2017), but despite decades of optical and nearinfrared (IR) studies, only a pair of candidate forming SSCs have been resolved in cold gas and dust emission (Oey et al. 2017; Turner et al. 2017). In both cases, CO (3-2) emission has been seen in association with an SSC in a starburst dwarf galaxy. This $\mathrm{CO}(3-2)$ emission may trace moderately more excited and dense gas than the CO (1-0) line.

In the Milky Way, approximately four massive protocluster candidates have been identified (e.g., Ginsburg et al. 2012; Fukui et al. 2016; Longmore et al. 2017; Urquhart et al. 2018), often following the criteria of Bressert et al. (2012). The Milky Way protoclusters have gas mass $\lesssim 10^{5} M_{\odot}$, somewhat lower than the extragalactic proto-SSC candidates. They appear likely to form $M_{\star} \sim 3 \times 10^{4} M_{\odot}$ clusters, assuming $\sim 30 \%$ efficiency (see Bressert et al. 2012).

In this article, we report the identification of 14 candidate proto-SSCs in NGC 253. This galaxy hosts one of the nearest nuclear starbursts $(d \sim 3.5 \mathrm{Mpc}$; Rekola et al. 2005), which 
produces stars at a rate of $\sim 2 M_{\odot} \mathrm{yr}^{-1}$ (e.g., Bendo et al. 2015; Leroy et al. 2015). This burst is fed by the galaxy's strong bar (Sorai et al. 2000), making NGC 253 a prototype for the common phenomenon of bar-fed nuclear starbursts (see Kormendy \& Kennicutt 2004).

Watson et al. (1996) and Kornei \& McCrady (2009) used the Hubble Space Telescope to discover a young ( $6 \mathrm{Myr}$ ) heavily extinguished $\left(A_{V} \sim 17 \mathrm{mag}\right) \mathrm{SSC}$ in the nuclear region of NGC 253. Any other SSCs in the nuclear region must be too heavily embedded to appear prominent in Hubble images, including the near-IR images presented in Walter et al. (2017). However, Ulvestad \& Antonucci (1997), following Turner \& Ho (1985), showed the presence of $\sim 30$ flat-spectrum compact $(\sim 1 \mathrm{pc})$ radio sources that could be embedded $\mathrm{H}$ II regions. One of these coincides with the SSC of Watson et al. (1996) and Kornei \& McCrady (2009).

Previous millimeter- and submillimeter-wave observations show that the NGC 253 nuclear region hosts massive, dense molecular clouds (Sakamoto et al. 2011; Leroy et al. 2015). The whole region resembles a heavily scaled-up version of the Milky Way's Central Molecular Zone (Sakamoto et al. 2011). Observations at $\theta \approx 0$." 5 resolution show that these clouds host compact $<10$ pc clumps of gas and dust, which have the appropriate masses to form massive clusters and are associated with signatures of massive star formation (Ando et al. 2017). Given these candidate SSC-forming structures and the presence of at least one known SSC, NGC 253 is the ideal target to catch SSC formation in the act.

In this article, we use the Atacama Large Submillimeter/ Millimeter Array (ALMA) to map dust emission from the NGC 253 starburst at $0 . \prime 11 \approx 1.9 \mathrm{pc}$ resolution, a factor of 5 improvement in linear scale (and a factor of 25 in area) compared to Ando et al. (2017). This allows us resolve individual forming SSCs, which have sizes of a few parsecs (e.g., see Portegies Zwart et al. 2010; Bressert et al. 2012; Longmore et al. 2014), at the heart of the Ando et al. (2017) clumps.

\section{Observations}

We used ALMA to observe NGC 253 at $\nu \sim 350 \mathrm{GHz}$ $(\lambda \sim 850 \mu \mathrm{m})$ as part of project 2015.1.00274.S (P.I. A. Bolatto). We observed NGC 253 with the main array in both an intermediate configuration and a $2 \mathrm{~km}$ extended configuration. We also used the $7 \mathrm{~m}$ array telescopes in the ALMA Compact Array (ACA) to recover short spacing information. The bandpass captures the submillimeter continuum from dust emission and covers several molecular rotational transitions, including $\mathrm{CO}(3-2), \mathrm{HCN}(4-3), \mathrm{HCO}^{+}(4-3), \mathrm{CS}(7-6)$, and $\mathrm{H}^{13} \mathrm{CN}(4-3)$. The full suite of line images and the extended molecular gas distribution and kinematics traced by CO (3-2) will be presented by N. Krieger et al. (2018, in preparation).

We combined the observatory-provided calibrated visibilities for two $12 \mathrm{~m}$ configurations and the ACA $7 \mathrm{~m}$ array and imaged them in version 5.1.1 of the Common Astronomy Software Application (CASA) using CASA's tclean task. The inclusion of the ACA means that scales out to $19^{\prime \prime}$ are recovered.

We are interested in the compact structures at the heart of the starburst. Therefore, when imaging the continuum, we adopted a Briggs robust parameter $r=-2$ (i.e., nearly uniform weighting). This weights the extended baselines more heavily to produce a higher resolution image. For the lines of interest in this article, $\mathrm{CS}(7-6)$ and $\mathrm{H}^{13} \mathrm{CN}(4-3)$, sensitivity remains a concern. Therefore, in the line images, we emphasized surface brightness sensitivity and used a standard Briggs weighting with robust parameter $r=0.5$.

After imaging, we convolved the continuum and line images to convert from an elliptical to a round beam shape. For the continuum image used in this article, the fiducial frequency is $\nu=350 \mathrm{GHz}$ and the final FWHM beam size is $\theta=0$ ". 11 . Before the convolution to a round beam, the beam of the continuum image has major and minor FWHM 0 ". $105 \times 0$ ". 065 .

The rms noise away from the source in the cleaned, round beam image is $0.2 \mathrm{~K}$ in Rayleigh-Jeans brightness temperature units, equivalent to $\approx 0.2 \mathrm{mJy}_{\text {beam }}{ }^{-1}$. For the $\mathrm{H}^{13} \mathrm{CN}(4-3)$ and CS (7-6) line images, the beam size is 0 " 175 (convolved from $\sim 0$." $14 \times 0$." 11 ) and the typical $\mathrm{rms}$ in the cube is $0.4 \mathrm{~K}$ per $5 \mathrm{~km} \mathrm{~s}^{-1}$ channel. The ancillary CO (3-2) and HCN (4-3) observations have similar resolution and noise. More details of the line imaging appear in N. Krieger et al. (2018, in preparation).

We compare the ALMA data to Karl G. Jansky's Very Large Array (VLA) imaging of $\nu=36 \mathrm{GHz}$ continuum emission (Gorski et al. 2017, and M. Gorski et al. 2018, in preparation). At this frequency and resolution, the radio continuum is likely to be predominantly free-free emission (e.g., see Murphy et al. 2011). These data have native resolution slightly better than that of the ALMA continuum image, with a FWHM beam of 0 " $096 \times 0$ ". 45 . We convolve them to the match the resolution of ALMA, $\theta=0$ ". 11, for analysis. After convolution, the VLA data have rms noise $\sim 0.03 \mathrm{mJy}_{\text {beam }}{ }^{-1}$.

We also compare the ALMA data to Hubble Space Telescope imaging of the near-IR $(\lambda=1.3 \mu \mathrm{m})$ continuum. These were obtained to serve as an off-line continuum measurement for the Paschen $\beta$ observations presented by Walter et al. (2017).

\section{Candidate Forming Super Star Clusters}

The top left panel in Figure 1 shows the whole disk of NGC 253 seen at $8 \mu \mathrm{m}$ by the Local Volume Legacy survey (Dale et al. 2009; Lee et al. 2009). The $8 \mu \mathrm{m}$ image shows the location of UV-heated small dust grains (likely polycyclic aromatic hydrocarbons) and so illustrates the overall morphology of the ISM in the galaxy. The bottom left panel zooms in on the square region indicated in the top panel. The square in the bottom left panel shows our regions of interest in this article. This is a square field, $10^{\prime \prime} \times 10^{\prime \prime}$ across, that includes most of the active star formation and dense clumps in the nuclear starburst. ALMA's 12 m antennas have a primary beam of $18^{\prime \prime}$ at $350 \mathrm{GHz}$, so the ALMA observations cover a somewhat larger field of view than we show in the Figure. In total, the nuclear burst in NGC 253 contains $\sim 3 \times 10^{8} M_{\odot}$ of molecular material and forms stars at $\sim 2 M_{\odot} \mathrm{yr}^{-1}$ (Bendo et al. 2015; Leroy et al. 2015).

\subsection{Dust Continuum and Gas}

The right panel of Figure 1 shows $\nu=350 \mathrm{GHz}$ $(\lambda \sim 855 \mu \mathrm{m})$ continuum emission from this inner region at $\theta=0$ ". $11 \approx 1.9 \mathrm{pc}$ resolution. At this frequency, thermal emission from large dust grains represents most of the 

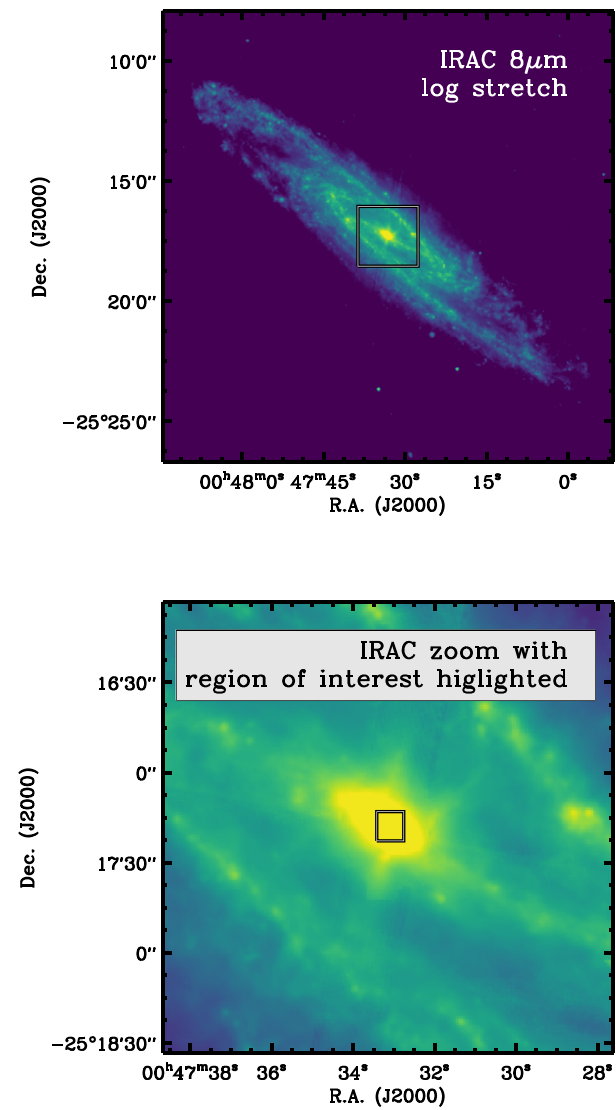
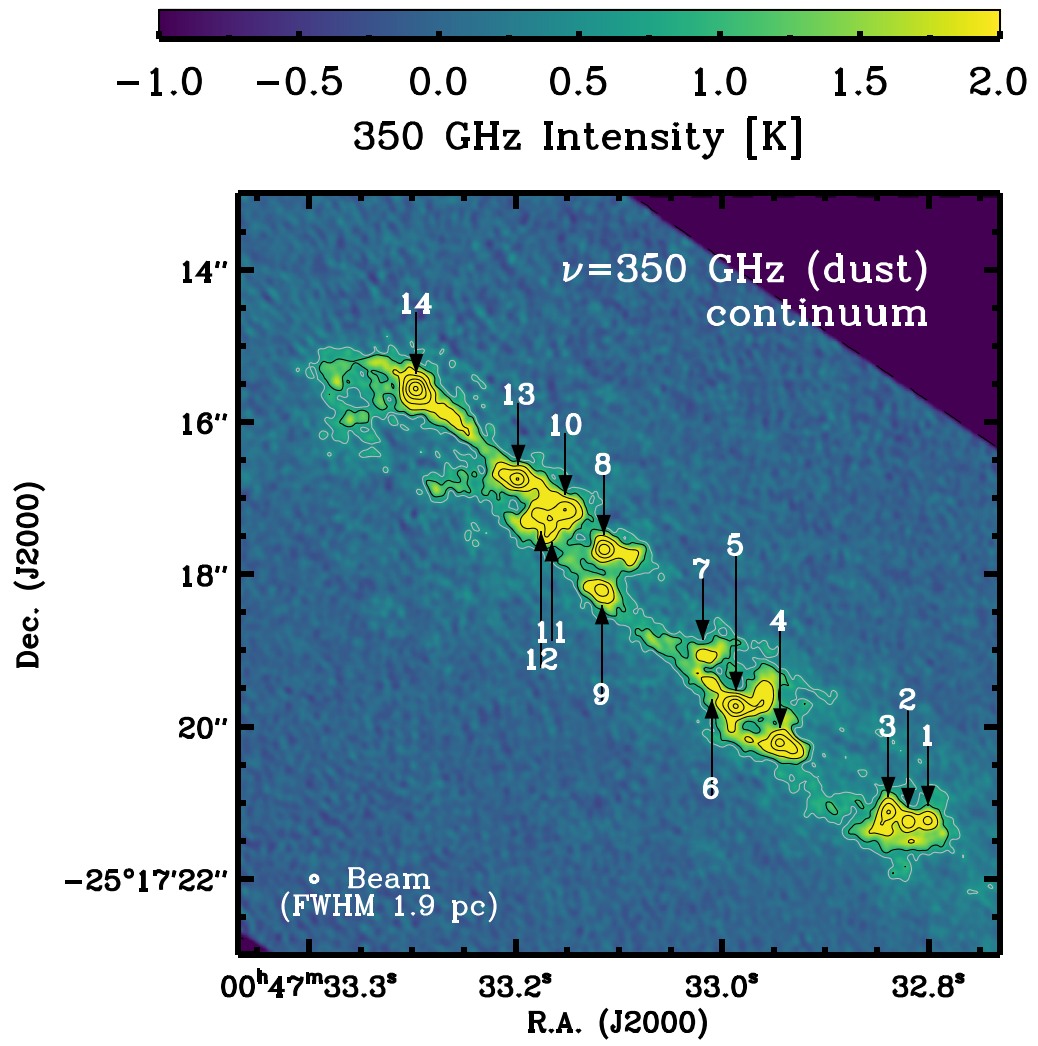

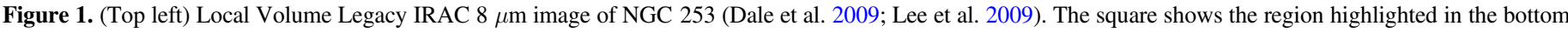

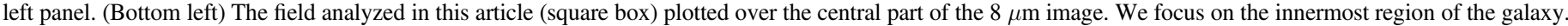

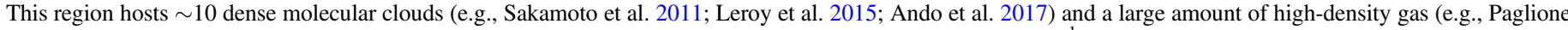

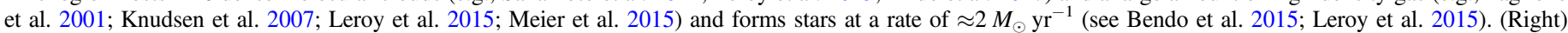

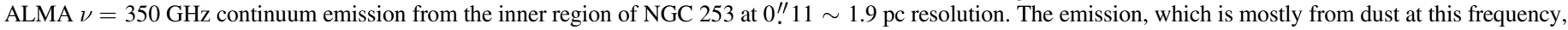

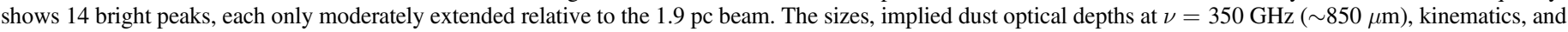

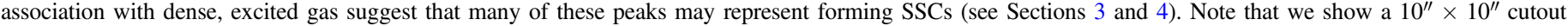

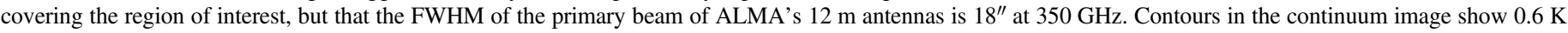
(gray), and $1,2,4, \ldots \mathrm{K}$ (black).

emission, with $350 \mathrm{GHz}$ in the Rayleigh-Jeans part of the spectral energy distribution (SED). In all but the most extreme conditions (which may include these peaks), this emission is optically thin. Thus, modulo temperature and emissivity variations, this emission offers an optically thin tracer of the column density distribution in the burst.

Our imaging reveals 14 bright, compact continuum peaks embedded in a network of extended emission with brightness temperatures of $T_{b} \sim 0.5-1 \mathrm{~K}$. We identify their locations using the local maximum finding routine from CPROPS (Rosolowsky \& Leroy 2006). This program finds peaks that (1) exceed all other pixel values within a square search kernel two times the synthesized beam across, and (2) are at least $5 \sigma$ above any contour shared with another peak, or $5 \sigma$ above $0 \mathrm{~K}$ if there is no shared contour.

These peaks have brightness of a few $\mathrm{K}$ up to a few tens of $\mathrm{K}$ and FWHM sizes of $\sim 2.5-4$ pc before any deconvolution. Thus, they appear bright and compact, but still are marginally resolved by our beam. As we will see, these sizes and the implied gas and dust masses of $\sim 10^{4}-10^{6} M_{\odot}$ suggest that these structures are forming SSCs.

The bright peaks are still associated with large surrounding reservoirs of gas. We show this in the top left panel of Figure 2, where we plot the line-integrated $\mathrm{CO}(3-2)$ intensity. The peaks sit at the hearts of the massive clouds and clumps studied by Sakamoto et al. (2011), Leroy et al. (2015), and Ando et al. (2017). They are not conspicuous in the integrated CO (3-2) intensity, although N. Krieger et al. (2018, in preparation) show that they can be identified from the CO kinematically.

The continuum peaks stand out in lines that trace highdensity molecular gas. The top right panel of Figure 2 shows our region of interest in line-integrated CS (7-6) as a color image with contours showing line-integrated $\mathrm{H}^{13} \mathrm{CN}(4-3)$ intensity. The $\mathrm{H}^{13} \mathrm{CN}(4-3)$ line emits most effectively at densities $n \gtrsim 10^{7} \mathrm{~cm}^{-3}$ (Shirley 2015) and $T \gtrsim 40 \mathrm{~K}$. The CS (7-6) emission, which also traces warm dense gas, has critical density of $\sim 3 \times 10^{7} \mathrm{~cm}^{-3}$ and requires $T \gtrsim 60 \mathrm{~K}$.

At coarser resolution, $\mathrm{HCN}(4-3)$ and $\mathrm{CS}(7-6)$ emission correlate with IR emission in star-forming galaxies, with a linear relationship relating IR and line luminosity (Zhang et al. 2014; Tan et al. 2018). Here we see $\mathrm{H}^{13} \mathrm{CN}(4-3)$ and $\mathrm{CS}$ (7-6) emission directly associated with the sites of massive star and cluster formation on $\sim 2$ pc scales. This direct association of these high density tracers with the sites of massive star formation fits in to a broader picture in which spectroscopic tracers of dense gas correlate with the rate of recent star formation (e.g., Gao \& Solomon 2004), with tracers of the densest gas showing the most linear correlations. 

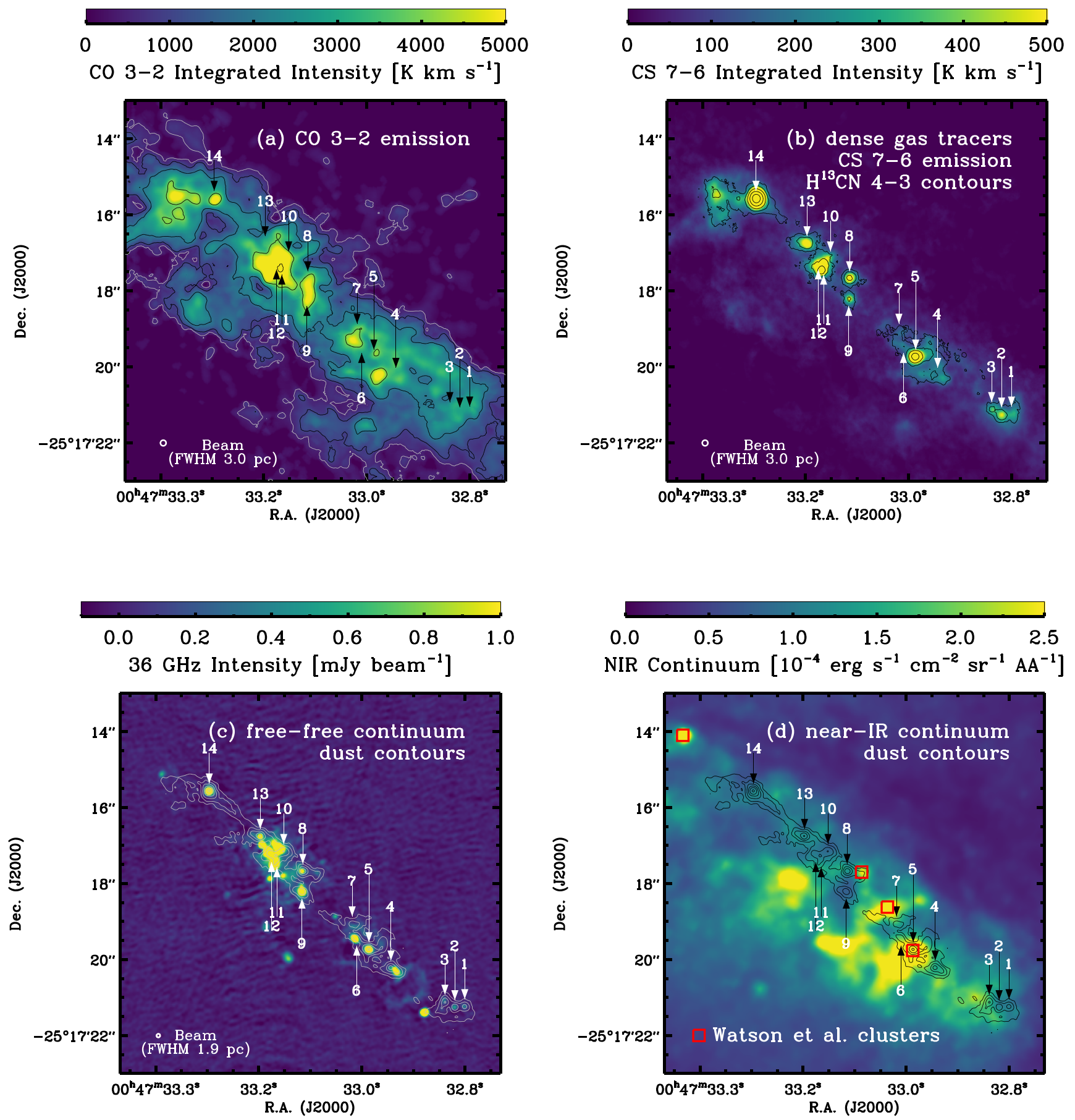

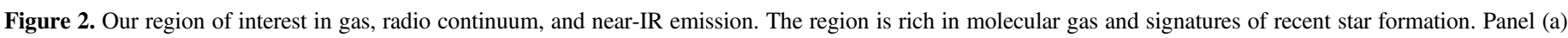

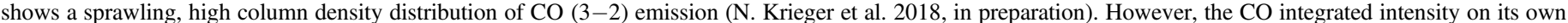

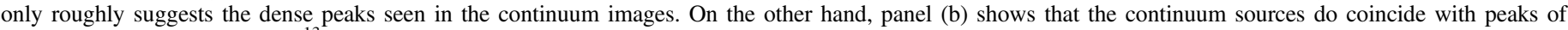

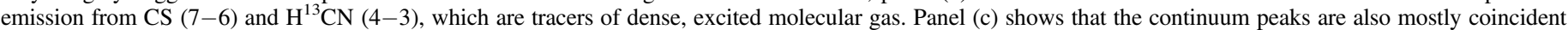

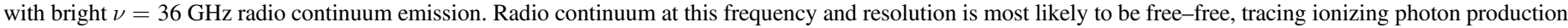

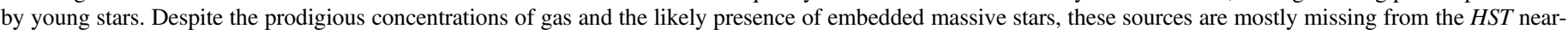

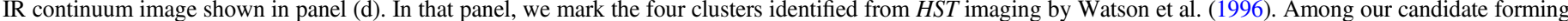

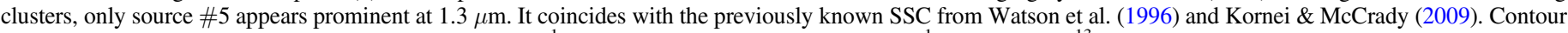

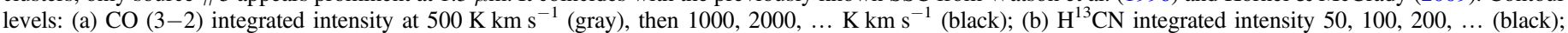
(c) ALMA $350 \mathrm{GHz}$ continuum image (i.e., Figure 1) at 1, 2, 4, ... K (gray); (c) ALMA $350 \mathrm{GHz}$ continuum image (i.e., Figure 1) at 1, 2, 4, ... K (black).

\subsection{Signatures of Massive Star Formation}

Are there actually signatures of young, massive stars associated with these peaks of gas and dust emission? The bottom left panel of Figure 2 shows $\nu \sim 36 \mathrm{GHz}$ continuum emission from our target field (Gorski et al. 2017, and
M. Gorski et al. 2018, in preparation) with the dust continuum contours overlaid. Our dust continuum peaks are coincident with, or near, peaks of bright radio continuum emission.

At this frequency and resolution, most of the sources in the $\nu=36 \mathrm{GHz}$ map arise from free-free emission. Through 
modulo loss of ionizing photons to dust and a mild dependence on the electron temperature and Gaunt factor, free-free emission directly traces ionizing photon production in a manner similar to that of optical recombination lines. However, unlike optical and near-IR recombination line emission, 36 $\mathrm{GHz}$ emission is almost totally unaffected by extinction. Thus, the bottom left panel of Figure 2 suggests that young, heavily embedded massive stars lie at or near most of our observed dust clumps.

The bottom right panel of Figure 2 shows that these signatures of massive star formation are almost totally obscured by dust even in the near-IR. We plot the near-IR $(1.3 \mu \mathrm{m})$ continuum as seen by Hubble's Wide Field Camera 3 (filter F130N). We also indicate the position of the four clusters identified by Watson et al. (1996) from earlier Hubble near-IR imaging. To match the astrometry of our near-IR data, which aligns well with the ALMA and VLA observations, we found it necessary to shift the measured positions from Watson et al. (1996) by $\Delta \alpha, \Delta \delta \approx+0$ "' 32, -0 ". 5 .

The image shows bright stellar continuum emission coincident with the brightest SSC known from Watson et al. (1996) and Kornei \& McCrady (2009). Otherwise, our dust peaks do not correspond to clear enhancements in the near-IR continuum. Given the presence of free-free emission, these sources are likely to be bright, compact, massive collections of young stars. However, the extinction in the inner region of the galaxy is too severe to pick them out even in the near-IR. This overwhelming extinction is striking, but not surprising. From the top left panel in Figure 2, we see that our peaks all lie at $I_{\mathrm{CO} 3-2} \gtrsim 2000 \mathrm{~K} \mathrm{~km} \mathrm{~s}^{-1}$. Under the conservative assumptions of thermalized $\mathrm{CO}$ lines, a low $\alpha_{\mathrm{CO}}=0.8 M_{\odot} \mathrm{pc}^{-2}\left(\mathrm{~K} \mathrm{~km} \mathrm{~s}^{-1}\right)^{-1}$, and a Galactic dust-to-gas ratio, this amount of gas still corresponds to $E(B-V) \sim$ $34 \mathrm{mag}$, or $A_{J} \sim 30 \mathrm{mag}$. Even without accounting for the dense concentrations within the clouds, the central region of NGC 253 is heavily extinguished and capable of hiding luminous clusters at near-IR wavelengths.

This $36 \mathrm{GHz}$ view of the NGC 253 nucleus resembles the $\sim 43 \mathrm{GHz}, 3$ pc resolution view of M82 by Tsai et al. (2009). In M82, another starburst at $d \sim 3.5 \mathrm{Mpc}$, Tsai et al. (2009) observed approximately nine compact continuum sources likely to be heavily embedded $\mathrm{H}$ II regions powered by massive clusters. Tsai et al. (2009) showed these compact H II regions to exist within dense gas structures observed at $\sim 45 \mathrm{pc}$ resolution. Based on our observations of NGC 253, it seems plausible, even likely, that some of the individual Tsai et al. (2009) sources will still be in the process of formation and that highresolution submillimeter observations of M82 would show associated parsec-scale concentrations of gas and dust. Schinnerer et al. (2007) observed similar coincidence of dense gas tracers and continuum signatures of embedded massive star formation at $\sim 10 \mathrm{pc}$ resolution in the inner region of NGC 6946, though there were some detailed differences between their HCN map and the NGC 6946 continuum emission seen by Tsai et al. (2006). Turner et al. (2003) and Turner \& Beck (2004) found similar compact H II regions surrounding the SSC in NGC 5253. Turner et al. (2017) showed CO emission from the same source, though that emission appears optically thin in $\mathrm{CO}$, perhaps indicating that the NGC 5253 cluster is at a later evolutionary stage than the ones that we observe.

\section{Properties of the Candidate Forming Super Star Clusters}

We estimate the size, line width, and fluxes associated with each peak and use these to gauge the masses of the candidate proto-SSCs in several ways.

\subsection{Size, Line Width, and Flux Measurements}

Size, peak temperature, and flux at $350 \mathrm{GHz}$. To measure the sizes associated with each peak, we build an azimuthally averaged radial profile centered on the peak. In each half-beam thick ring centered on the peak, we calculate the median intensity. Using the median suppresses the influence of nearby peaks and the bright surrounding filamentary features, and so emphasizes the profile of the central peak. We further reject $3 \sigma$ outliers about this median profile. Figure 3 shows the resulting profiles appear as black points, with error bars showing the scatter about the profile. Blue lines show a Gaussian fit to these profiles; this fit includes a background term, which is small in all cases.

To compute deconvolved sizes, we subtract the beam size in quadrature from the Gaussian fit to the profile. We also correct the peak temperature for the effects of the beam by scaling the measured peak temperature by the ratio of measured source area to the deconvolved source area. The deconvolved profiles appear as red lines in Figure 3.

We report the measured sizes in Table 1. As a check, we also fit two-dimensional Gaussians to each source. The deconvolved FWHM size from the Gaussian fits agree with our measured sizes with a scatter of $\pm 0.3 \mathrm{pc}$. We adopt this as our uncertainty on the size, with the uncertainty dominated by the choice of method. We take the fractional uncertainty in the deconvolved $T_{\text {peak }}$ to be the sum in quadrature of the fractional uncertainty due to statistical noise and the fractional uncertainty in the deconvolved beam area.

From the fits to the profiles, we also calculate the flux of each source at $\nu=350 \mathrm{GHz}$, which we give in Table 1 . The statistical uncertainties on this flux are low, because the peaks are all detected at high signal-to-noise ratio $(\mathrm{S} / \mathrm{N})$. In this case, we quote a $10 \%$ uncertainty on the overall flux for each source, reflecting a mixture of calibration uncertainty (which should be covariant among all sources), uncertainty in the image reconstruction, and uncertainty due to the adopted method.

For reference, from a lower-resolution, robust-weighted version of the continuum map, we calculate a total $350 \mathrm{GHz}$ flux of $\approx 1.9 \mathrm{Jy}$ by integrating all emission above $\mathrm{S} / \mathrm{N}=3$. The sources in Table 1 have total flux $0.28 \mathrm{Jy}$, and so account for $\sim 15 \%$ of the total $350 \mathrm{GHz}$ emission from the nuclear region.

Line widths. We measure the line width associated with each peak. To do this, we define a series of apertures. The aperture associated with a peak has radius equal to the FWHM fit (not deconvolved) size of the peak and sits centered on the peak. The background region associated with each aperture extends from the radius one to three times the FWHM fit size of the central source. The background excludes apertures associated with other peaks. We calculate the source spectrum by subtracting the average spectrum in the background from the average spectrum in the aperture. Note that the central apertures that we use are never less than 0." 22 across (diameter). Thus, the measurement region is always at least moderately extended compared to the 0 !. 175 beam of the $\mathrm{H}^{13} \mathrm{CN}(4-3)$ and $\mathrm{CS}(7-6)$ cubes. 

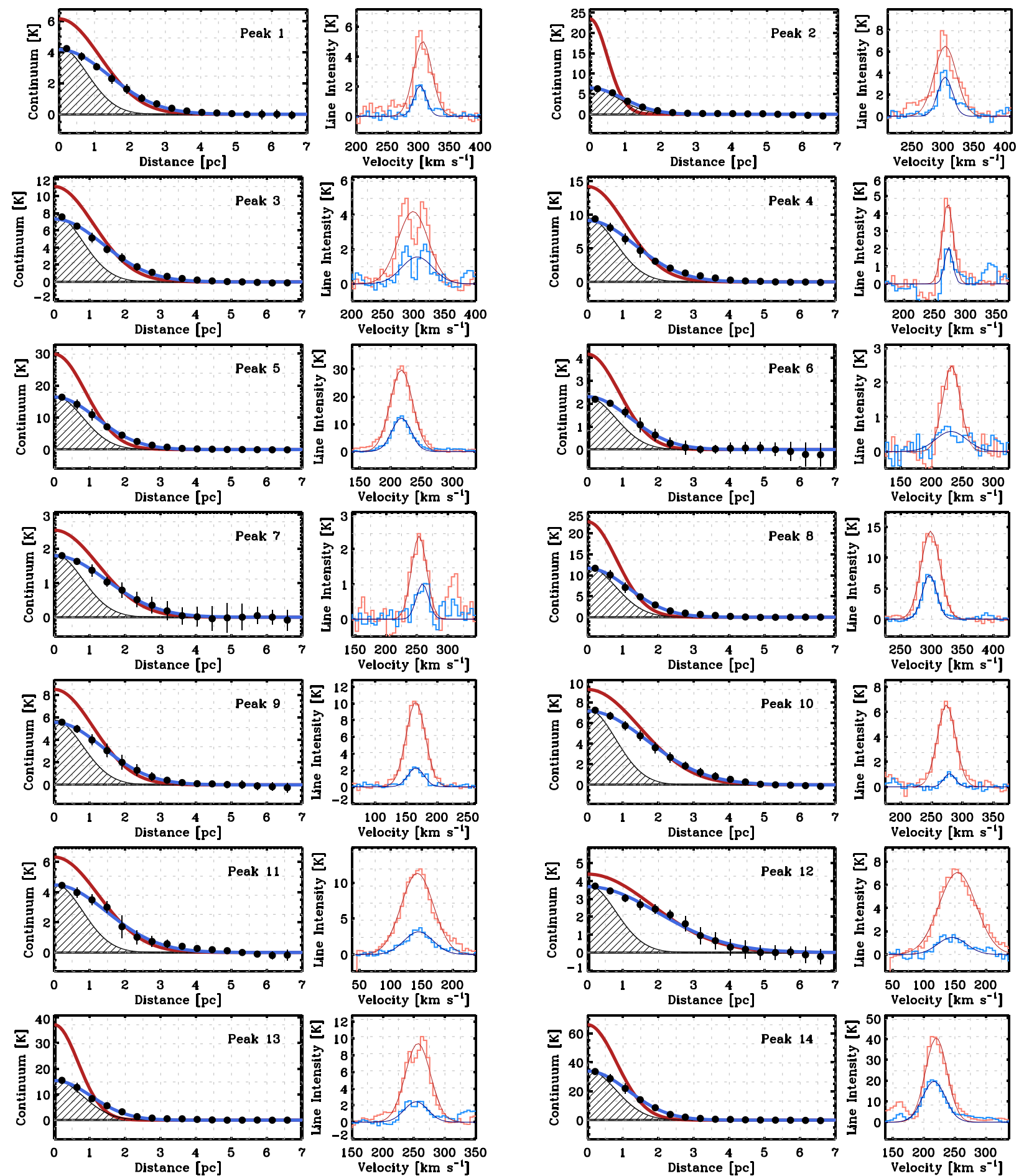

Figure 3. Spatial (left) and spectral (right) profiles of our 14 peaks. The left column shows the binned median-based radial profile of $350 \mathrm{GHz}$ emission about each peak (black bins, with a blue Gaussian fit). The black, shaded profile in each panel indicates the shape of the synthesized beam. The red profile shows the inferred shape of the peak after deconvolving the beam. The spectra show the background-subtracted CS (7-6) emission in red and $\mathrm{H}^{13} \mathrm{CN}(4-3)$ emission in blue. Lines indicate Gaussian fits to the profiles. Spectrum \#3 shows a split line profile, indicative of a shell geometry, self-absorption, or substructure.

We fit a Gaussian to the background-subtracted for CS (7-6) and $\mathrm{H}^{13} \mathrm{CN}(4-3)$ spectra, fitting over a velocity range picked by eye to cover the emission line. Figure 3 shows both background-subtracted spectra and the fits for each peak. We take the linear average of the two line widths as the characteristic line width for the source. We adopt one half the difference in the line width derived between the two lines as our uncertainty.
$36 \mathrm{GHz}$ fluxes. We measure fluxes for each source from the VLA $36 \mathrm{GHz}$ map. To do this, we subtract the average intensity in the local background region from the region inside the aperture. Then we sum the flux inside the aperture. We use the same apertures used to derive the line widths.

Similar to the case of the ALMA fluxes, the statistical uncertainty in the $36 \mathrm{GHz}$ flux is small (compare the fluxes in Table 1 to the $0.02 \mathrm{mJy}_{\text {beam }}{ }^{-1}$ noise). We adopt an 
Table 1

Measured Properties Candidate Young Clusters in NGC 253

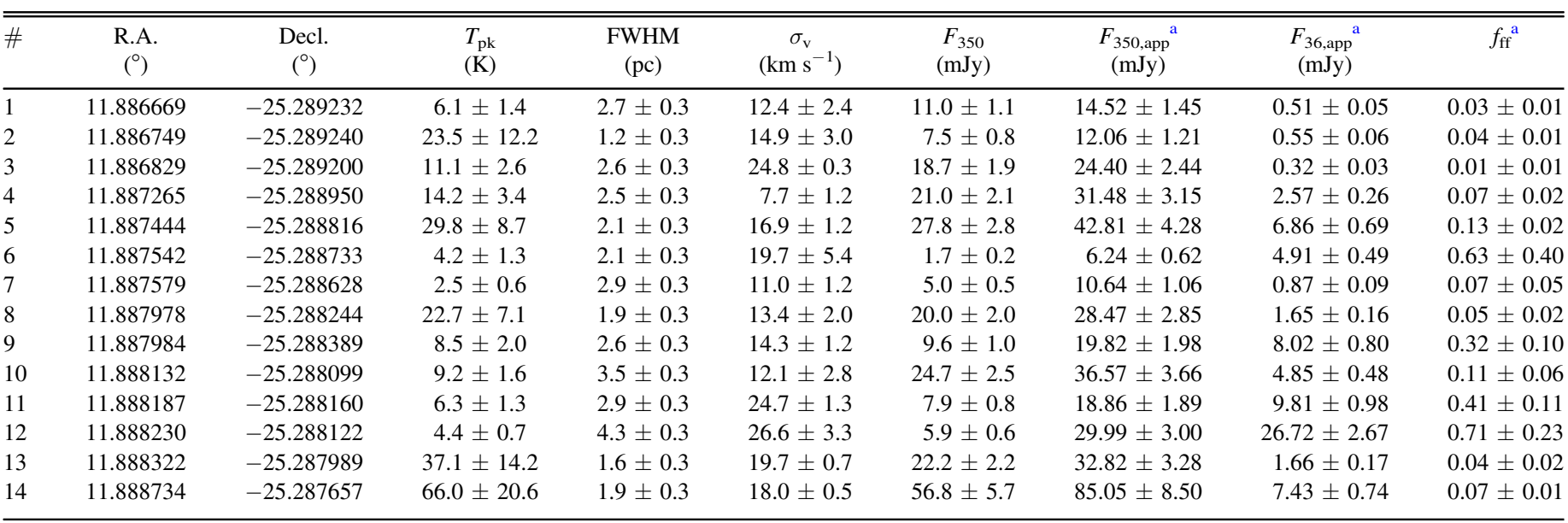

Notes. R.A., decl. refer to peak position in the $350 \mathrm{GHz}$ continuum image. FWHM size assumes a distance of $3.5 \mathrm{Mpc}$ and already accounts for the deconvolution of the 0 . $11 \approx 1.9 \mathrm{pc}$ beam. $T_{\mathrm{pk}}$ reports the peak intensity, after deconvolving the beam, at $350 \mathrm{GHz}$ in Rayleigh-Jeans brightness temperature units. $\sigma_{v}$ is the linear average of the $\mathrm{CS}(7-6)$ and $\mathrm{H}^{13} \mathrm{CN}(4-3)$ rms line width. $F_{350}$ is the flux at $350 \mathrm{GHz}$ estimated from the Gaussian fit to the profile. $F_{36}$ is the flux of $36 \mathrm{GHz}$ emission obtained from aperture photometry, this is scaled into a luminosity using the distance and used to estimate $Q_{0}$ and $M_{\star}$ using the equations in the text. $f_{\mathrm{ff}}$ refers to the estimated fractional free-free contribution to the $350 \mathrm{GHz}$ emission based on comparing fluxes measured in matched apertures.

${ }^{a}$ Values measured in apertures centered on the peaks. The apertures have radius equal to the FWHM size of the source before deconvolution (i.e., to recover this add $1.9 \mathrm{pc}$ in quadrature to the value in the table). See the text for more details.

uncertainty of $10 \%$ to reflect calibration and image reconstruction uncertainties.

Based on $36 \mathrm{GHz}$ emission, we estimate the fractional contribution of free-free flux to the ALMA band via:

$$
f_{\mathrm{ff}} \approx\left(\frac{36}{350}\right)^{0.1} \frac{F_{36}}{F_{350}}
$$

where the first factor reflects the expected -0.1 spectral index from optically thin free-free emission and $F_{36}$ and $F_{350}$ refer to the observed total flux at $36 \mathrm{GHz}$ and $350 \mathrm{GHz}$. For this application only, we measure fluxes from the ALMA $350 \mathrm{GHz}$ map in exactly the same way that we measure the $36 \mathrm{GHz}$ fluxes (i.e., using aperture photometry and the same aperture definitions). We report both sets of fluxes in Table 1.

$f_{\text {ff }}$ is the fraction of the $350 \mathrm{GHz}$ flux in the aperture that can be attributed to free-free emission, assuming that all of the $36 \mathrm{GHz}$ flux comes from optically thin free-free emission. A value $\ll 1$ is expected if thermal dust emission makes a large contribution to the emission from the $350 \mathrm{GHz}$ band. A value $\sim 1$ indicates either that free-free emission contributes a large fraction of the $350 \mathrm{GHz}$ emission or that the $36 \mathrm{GHz}$ emission is not free-free in nature (expected, e.g., if synchrotron contributes heavily).

We see high $f_{\mathrm{ff}}$ around four sources: peaks \#6 $\left(f_{\mathrm{ff}}=0.63\right)$, \#9 (0.32), \#11 (0.41), and \#12 (0.71). With only two bands, we cannot distinguish between contamination of the $350 \mathrm{GHz}$ band by free-free or the $36 \mathrm{GHz}$ band by synchrotron. Additional high-resolution measurements at $\sim 100-200 \mathrm{GHz}$ and at $\sim 1-25 \mathrm{GHz}$ will help resolve the nature of the emission (some observations at slightly coarser resolution already exist Ulvestad \& Antonucci 1997; Mohan et al. 2005).

Peak \#6 is indeed weak in the ALMA map but is a clear point source in the $36 \mathrm{GHz}$ map. This may represent a cluster in a later stage of evolution or a supernova ( $\mathrm{SN}$ ) remnant. The other three sources lie near the galaxy nucleus. Peaks \#11 and \#12 lie in a region where Mohan et al. (2005) do see substantial radio recombination line flux, but also a complex structure. Those authors speculate that the nucleus, which is $\sim 10 \mathrm{pc}$ away, might contribute to ionization in the region. In any case, we apply $f_{\mathrm{ff}}$ as a correction to the gas mass estimates, viewing this as the most conservative option.

\subsection{Resemblance to a Known Milky Way Protocluster}

As a check, we construct profiles similar to those in Figure 3 for the known Galactic protoclusters Sgr B2. This pair of bright sources near the Galactic center is regarded as very likely to be forming young massive clusters (see Ginsburg et al. 2018; Urquhart et al. 2018, and references therein). We degrade the ATLASGAL $500 \mu \mathrm{m}$ data to a resolution of $1.9 \mathrm{pc}$ (FWHM) and scale the intensity assuming a spectral index of 4 (i.e., optically thin dust with $\beta=2$ ). We also extract a spectrum of $\mathrm{HC}_{3} \mathrm{~N}(24-23)$ at $3 \mathrm{pc}(\mathrm{FWHM})$ resolution to serve as a proxy for our $\mathrm{H}^{13} \mathrm{CN}$ and $\mathrm{CS}$ measurements, though we note that $\mathrm{HC}_{3} \mathrm{~N}(24-23)$ has larger excitation requirements than does the $\mathrm{H}^{13} \mathrm{CN}(4-3)$ or $\mathrm{CS}(7-6)$.

The resulting profile and spectrum, shown in Figure 4, show that Sgr B2 would have a slightly larger size and narrower line width than our candidate clusters. It would also have among the lowest brightness temperatures of our sources. Overall, however, the structure in Figure 4 does resemble what we see for our NGC 253 sources (Figure 3). The comparison gives us confidence that we detect moderately more compact, scaledup versions of a known Galactic protocluster.

Sgr B2 appears as a single extended source in this exercise, but also breaks into two massive protoclusters at higher resolution (e.g., see Figure 1 in Ginsburg et al. 2018). Therefore, this comparison also highlights the likelihood that, despite our high (for extragalactic work) $1.9 \mathrm{pc}$ resolution, some of our sources may break into two or more smaller, more compact protoclusters when observed at higher resolution. According to a first look at ALMA observations that are approximately two times higher resolution obtained during 

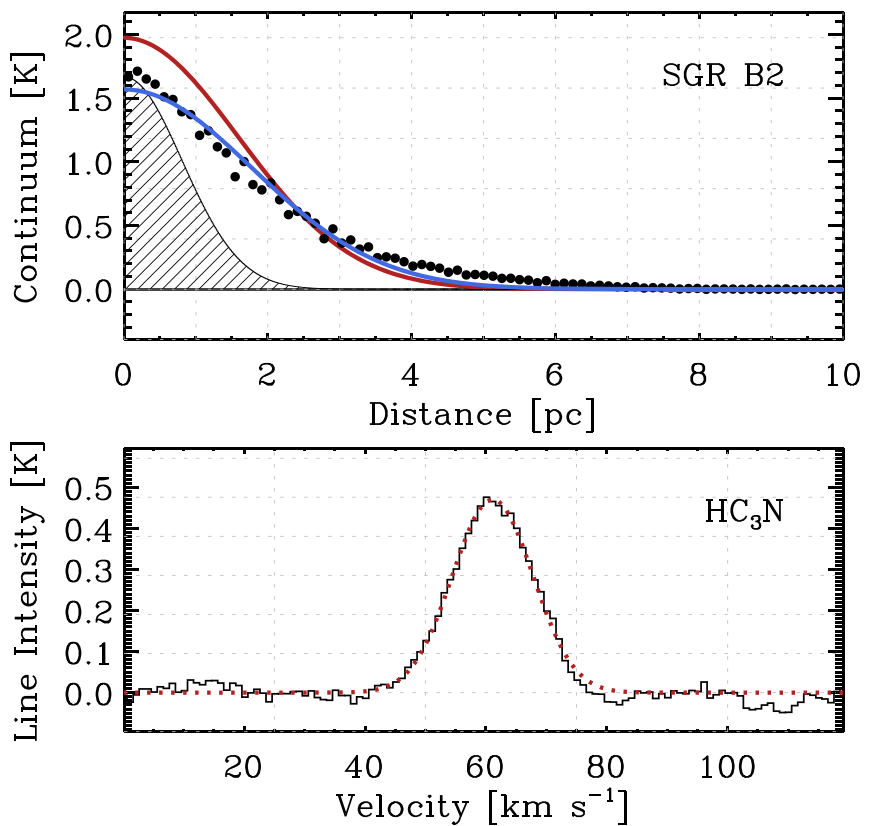

Figure 4. Profile similar to those in Figure 3 but for the known Milky Way protoclusters in Sgr B2 (e.g., see Ginsburg et al. 2018). We show the profile of the sources as seen by ATLASGAL (see Urquhart et al. 2018) matched to our $1.9 \mathrm{pc}$ resolution (top). On the bottom, we show a spectrum of $\mathrm{HC}_{3} \mathrm{~N}(24-23)$. In both cases, we have plotted the data similar to how we show our profiles, removing a local background from the radial profile and subtracting the continuum from the spectrum. The combined Sgr B2 protoclusters show peak continuum brightness $\sim 2 \mathrm{~K}$, FWHM extent $\sim 3.7 \mathrm{pc}$, and $1 \sigma$ line width $\sim 6.7 \mathrm{~km} \mathrm{~s}^{-1}$. Thus, they would appear as among the least bright and least compact of our sources, with the narrowest line widths. However, they do overall resemble the sources that we see in NGC 253, so that our observations pick out sources that resemble scaled up versions of known protoclusters in the Milky Way. Similar to Sgr B2, we might expect some of our sources to break up into two or more protoclusters within our $1.9 \mathrm{pc}$ beam.

review of this article, many of our sources do resolve into several smaller structures at higher resolution. In almost all cases, though, a single bright source still contributes most of the submillimeter flux. Although we might expect the sizes of our sources to shrink some and to find some nearby lower mass clusters, we expect our main results to hold with improved resolution.

\subsection{Gas, Stellar, and Dynamical Masses}

Based on their size, line width, and fluxes, we estimate the gas, stellar, and dynamical masses of these cluster candidates. We report these in Table 2. To do this, we assume that (1) the free-free corrected $350 \mathrm{GHz}$ emission arises from dust with some adopted temperature and emissivity, which we take to be well mixed with the gas with some characteristic dust-to-gas ratio; (2) the $36 \mathrm{GHz}$ represents free-free emission emitted by a young stellar population on the zero age main sequence (ZAMS); and (3) our objects are in virial equilibrium, so that their sizes and line widths together indicate their dynamical mass.

\subsubsection{Zero Age Main-sequence Stellar Mass}

Assuming that all of the $36 \mathrm{GHz}$ emission is produced by free-free interactions, we can estimate the ionizing photon production rate of each source. From this, we can calculate the mass of ZAMS stars needed to produce this number of ionizing photons.
Following Murphy et al. (2011) and Caplan \& Deharveng (1986), a $36 \mathrm{GHz}$ luminosity, $L_{36}$, implies an ionizing photon production rate of:

$$
Q_{0} \sim 1.06 \times 10^{26} L_{36} \mathrm{~s}^{-1}
$$

We have assumed an electron temperature $T_{e}=7000 \mathrm{~K}$ (slightly higher than the estimate for NGC 253 by Bendo et al. 2015). Here, $Q_{0}$ is the ionizing photon production rate per second and $L_{36}$ is measured in erg s${ }^{-1} \mathrm{~Hz}^{-1}$.

Based on Starburst99 calculations (Leitherer et al. 1999), for a ZAMS population the mass $\left(M_{\star}\right)$ relates to the ionizing photon production rate $\left(Q_{0}\right)$ via

$$
M_{\star} \sim \frac{Q_{0}}{4 \times 10^{46}} M_{\odot}
$$

We arrive at this value by simulating $10^{6} M_{\odot}$ single stellar population, with the initial mass function (IMF) of Kroupa (2001), a maximum stellar mass of $100 M_{\odot}$, and the default stellar evolution tracks and tuning parameters. Then we divide the ionizing photon output at time zero by mass of the stellar population. Together, Equations (3) and (2) yield the mass of the embedded stellar population needed to produce the observed $36 \mathrm{GHz}$ flux via free-free emission.

Our candidate proto-SSCs have median $\log _{10} M_{\star}\left[M_{\odot}\right]=5.1$ and range $\log _{10} M_{\star}\left[M_{\odot}\right] \sim 4.1-6.0$. According to this calculation, all of our sources already qualify as young massive clusters (i.e., $M_{\star} \gtrsim 10^{4} M_{\odot}$ Portegies Zwart et al. 2010; Longmore et al. 2014).

Note that the two highest values, for peaks \#11 and \#12, should be regarded with suspicion because of the high $f_{\mathrm{ff}}$ found for these objects (Table 1) and the uncertain nature of the ionization in this region (see above).

Our $M_{\star}$ is a linear translation of the $36 \mathrm{GHz}$ flux, and our sources are detected at high $\mathrm{S} / \mathrm{N}$. This yields small statistical uncertainties, $\sim \pm 10 \%$, and systematic effects dominate the uncertainty in $M_{\star}$. First, uncertainties in the Gaunt factor imply a systematic uncertainty of $\approx \pm 20 \%$ (Rybicki \& Lightman 1986). Second, if dust absorbs a significant fraction of the ionizing photons, our $M_{\star}$ will represent an underestimate. Loss of ionizing photons to dust already appears to be a significant effect in massive star-forming regions in the Milky Way (Binder \& Povich 2018), so we do expect this to also be important in the dustier, dense nucleus of NGC 253, but the magnitude of the effect is not clear. Furthermore, if there is ongoing accretion and many of the stars in the cluster have not yet reached the main sequence, we would also expect a higher mass per ionizing photon produced. Most of these effects have the sense that our quoted $M_{\star}$ likely represents a moderate underestimate. The calculation also has the usual uncertainties related to the upper mass cutoff and shape of the IMF. Our estimates also take no account of the influence of binary stars (e.g., Eldridge et al. 2017). Note that, following Xiao et al. (2018), we expect the inclusion of binaries to have the largest effect after a few megayears. Thus we expect binarity to be a minor concern for this article, which focuses on young sources. Finally, note that we estimate ZAMS mass in an aperture centered on the cluster. Future high-resolution comparison of the $36 \mathrm{GHz}$ structure, dust, and gas will help us understand how much of this mass is, in fact, directly associated with the gas and dust peaks. 
Table 2

Estimated Physical Properties Candidate Young Clusters in NGC 253

\begin{tabular}{|c|c|c|c|c|c|c|c|c|}
\hline \# & $\begin{array}{c}\log _{10} M_{\mathrm{VT}}{ }^{\mathrm{a}} \\
\left(M_{\odot}\right)\end{array}$ & $\begin{array}{c}\log _{10} M_{\mathrm{gas}}{ }^{\mathrm{b}} \\
\left(M_{\odot}\right)\end{array}$ & $\begin{array}{c}\log _{10} M_{\star}{ }^{\mathrm{c}} \\
\left(M_{\odot}\right)\end{array}$ & $\begin{array}{c}\log _{10} \Sigma_{\mathrm{gas}+\star^{\mathrm{b}, \mathrm{c}}} \\
\left(M_{\odot} \mathrm{pc}^{-2}\right)\end{array}$ & $\begin{array}{c}\log _{10} \rho_{\text {gas }+\star}{ }^{\mathrm{b}, \mathrm{c}} \\
\left(M_{\odot} \mathrm{pc}^{-3}\right)\end{array}$ & $\begin{array}{c}\log _{10} \tau_{\mathrm{ff}}^{\mathrm{b}, \mathrm{c}} \\
(\mathrm{yr})\end{array}$ & $\begin{array}{c}p_{r} / M_{\star} \\
\left(\mathrm{km} \mathrm{s}^{-1}\right)\end{array}$ & $\begin{array}{c}v_{\mathrm{esc}} \\
\left(\mathrm{km} \mathrm{s}^{-1}\right)\end{array}$ \\
\hline 1 & 5.6 & 4.9 & 4.3 & 3.9 & 3.4 & 5.2 & 79.4 & 13.2 \\
\hline 2 & 5.4 & 4.7 & 4.3 & 4.6 & 4.4 & 4.7 & 64.7 & 18.2 \\
\hline 3 & 6.2 & 5.1 & 4.1 & 4.1 & 3.7 & 5.1 & 443.8 & 16.7 \\
\hline 4 & 5.1 & 5.1 & 5.0 & 4.4 & 3.9 & 4.9 & 18.6 & 22.0 \\
\hline 5 & 5.7 & 5.3 & 5.4 & 4.8 & 4.5 & 4.7 & 20.1 & 33.2 \\
\hline 6 & 5.9 & 3.6 & 5.3 & 4.5 & 4.1 & 4.9 & 0.8 & 21.8 \\
\hline 7 & 5.5 & 4.5 & 4.5 & 3.7 & 3.2 & 5.3 & 17.9 & 10.7 \\
\hline 8 & 5.5 & 5.2 & 4.8 & 4.6 & 4.2 & 4.8 & 51.3 & 23.3 \\
\hline 9 & 5.7 & 4.7 & 5.5 & 4.5 & 4.1 & 4.9 & 3.5 & 26.5 \\
\hline 10 & 5.7 & 5.2 & 5.3 & 4.3 & 3.7 & 5.1 & 17.1 & 22.4 \\
\hline 11 & 6.2 & 4.5 & 5.6 & 4.5 & 4.0 & 4.9 & 3.5 & 26.8 \\
\hline 12 & 6.4 & 4.1 & 6.0 & 4.6 & 3.9 & 5.0 & 0.5 & 35.3 \\
\hline 13 & 5.7 & 5.2 & 4.8 & 4.8 & 4.5 & 4.6 & 89.4 & 27.4 \\
\hline 14 & 5.7 & 5.7 & 5.5 & 5.1 & 4.8 & 4.5 & 53.3 & 45.5 \\
\hline
\end{tabular}

Notes. Masses estimated following Section 4.3. The uncertainty in all of the mass estimates are dominated by systematic uncertainties. We note the dominant uncertainty for each quantity in the associated footnote. $M_{\mathrm{VT}}$ refers to the dynamical mass estimated from the virial theorem. $M_{\mathrm{gas}}$ refers to gas mass estimated from dust emission at $350 \mathrm{GHz} . M_{\star}$ refers to the ZAMS stellar mass needed to match the ionizing photon production rate estimated from the $36 \mathrm{GHz}$ emission. $\Sigma_{\text {gas }+\star}$ is the estimated gas plus stellar surface density within the two-dimensional FWHM given our size and mass estimates. $\rho_{\text {gas }+\star}$ is mass volume density within the threedimensional FWHM given our given our size and mass estimates. $\tau_{\mathrm{ff}}$ is the gravitational freefall time implied by that density. $p_{r} / M_{\star}$ is the equivalent radial momentum per unit stellar mass calculated from the gas velocity dispersion, gas mass, and stellar mass (Equation (11)).

${ }^{a}$ Dynamical mass from the virial theorem. Dominant uncertainty is sub-beam structure, including whether the source breaks up into multiple smaller sources; $\sim 0.3$ dex systematic uncertainty is plausible with our estimates likely to be high. Statistical uncertainty $\approx 0.1$ dex.

${ }^{\mathrm{b}}$ Gas-mass based quantities. Uncertainties from the assumed dust temperature, dust-to-gas ratio, and opacity. Likely magnitude is $\sim 0.4-0.5$ dex with our estimates likely to be low.

${ }^{c}$ ZAMS stellar mass needed to produce the observed $36 \mathrm{GHz}$ emission as free-free following Equations (2) and (3). Mild uncertainty due to the assumed temperature, Gaunt factor, and possible contamination by synchrotron. Larger uncertainties regarding the amount of ionizing photons absorbed by dust and the possibility of premain sequence stars in the source.

\subsubsection{Implications for the Ionized Gas Content}

From $Q_{0}$ and a plausible size, we estimate the ionized gas mass and density associated with each source. We posit an H II region with radius $r_{S}$ at the heart of each source. Assuming Case $\mathrm{B}$ recombination, complete ionization of hydrogen, and 1.36 contribution of helium by mass, we expect

$$
\begin{aligned}
M_{\mathrm{ion}} & \approx\left(\frac{Q_{0}}{\alpha_{\mathrm{B}}} 4 / 3 \pi r_{S}^{3}\right)^{0.5} 1.36 m_{\mathrm{H}} \\
M_{\mathrm{ion}} & \approx 684 M_{\odot}\left(\frac{Q_{0}}{10^{51} \mathrm{~s}^{-1}}\right)^{0.5}\left(\frac{r_{S}}{1 \mathrm{pc}}\right)^{1.5} .
\end{aligned}
$$

Here, $\alpha_{B} \approx 3.4 \times 10^{-13} \mathrm{~cm}^{3} \mathrm{~s}^{-1}$ is the adopted recombination rate coefficient corresponding to an $\mathrm{H}$ II region temperature of $7000 \mathrm{~K}$ (Draine 2011). A higher ionizing photon flux requires more ionized gas to be present, and a larger $\mathrm{H}$ II region implies more ionized gas mass. The order of magnitude for our $M_{\text {ion }}$ agrees with the calculations by Ulvestad \& Antonucci (1997), though the adopted distances and other details do vary.

Measuring the sizes of the $\mathrm{H}$ II regions will help constrain this measurement and is a natural next direction. For $r_{S} \sim 1 \mathrm{pc}$, ionized gas contributes appreciably only to source \#6 $\left(M_{\text {ion }} / M_{\text {gas }} \sim 50 \%\right.$ for our fiducial assumptions), source \#11 $(\sim 10 \%)$, and source \#12 ( 40\%). In all other cases, the fractional contribution of ionized gas to the gas mass is $<10 \%$ and usually $\lesssim 1 \%$. Again, this implies that sources \#11 and \#12, which lie near the nucleus, need more detailed study (see also Mohan et al. 2005).
The densities implied by this calculation appear reasonable.

$$
n_{\mathrm{ion}} \approx 4850 \mathrm{~cm}^{-3}\left(\frac{Q_{0}}{10^{51} \mathrm{~s}^{-1}}\right)^{0.5}\left(\frac{r_{S}}{1 \mathrm{pc}}\right)^{-1.5}
$$

yields densities mostly in the range $n_{\text {ion }} \sim 10^{3}-10^{4} \mathrm{~cm}^{-3}$ for $r_{S}=1 \mathrm{pc}$, but this depends strongly on $r_{S}$. A measured value of $r_{S}$ will allow us to determine whether the $\mathrm{H}$ II regions are overpressured and evolving (and, e.g., to compare to Krumholz \& Matzner 2009) or in approximate pressure equilibrium with the surrounding gas (e.g., as in the center of IC 342 Meier et al. 2011).

\subsubsection{Gas Mass from Dust}

We estimate the mass of gas associated with each protocluster candidate from the $350 \mathrm{GHz}$ dust emission. To do this, we estimate the optical depth at the peak by contrasting the measured brightness with an estimate of the true dust temperature. Then we convert the optical depth to a dust column using an assumed mass absorption coefficient. We convert the dust to a gas column via an adopted dust-to-gas ratio. Finally, we scale the gas column at the center of the proto-SSC by the area of the peak to calculate a total gas mass. In the future, we hope to constrain the dust-to-gas ratio by comparing our dust mass estimates to gas mass estimates based on the gas emission lines. At the moment, we consider the dust a more reliable estimate of the gas content than the molecular lines that we observe.

We assume a fiducial temperature of $T_{\text {dust }}=130 \mathrm{~K}$, assuming that the clusters coincide with the warm component seen in 
ammonia spectroscopy (Mangum et al. 2013; Gorski et al. 2017 ) and that the gas and dust are collisionally coupled. We convert our measured $350 \mathrm{GHz}$ intensity at the peak, $I_{350}$, to a dust optical depth via

$$
I_{350}=\left[1-\exp \left(-\tau_{350 \mathrm{GHz}}\right)\right] B_{\nu}\left(T_{\text {dust }}\right) .
$$

Here, $I_{350}$ is our measured $350 \mathrm{GHz}$ intensity, corrected for free-free contamination using the value in Table 1 , and expressed in cgs units. $B_{\nu}$ is the Planck function evaluated at $350 \mathrm{GHz}$ for our adopted dust temperature. This formulation deals better with mild optical depth effects than assuming the emission to be optically thin. However, if the emission is strongly clumped within our beam, then these optical depth effects will be underestimated.

Equation (6) yields optical depths at $350 \mathrm{GHz}$, mostly in the range of 0.035 to 0.35 , with $\tau_{350 \mathrm{GHz}} \sim 0.09$ on average. The dust appears to be moderately optically thin at $350 \mathrm{GHz}$. Because of the frequency-dependent opacity of dust, $\tau_{\nu} \propto \nu^{\beta}$ with $\beta \sim 1.5-2$, these values imply that these sources will be quite optically thick at higher frequencies (shorter wavelengths) where most of the energy is emitted.

After calculating $\tau$, we convert to a dust mass surface density using an assumed mass absorption coefficient, $\kappa$. We adopt $\kappa=1.9 \mathrm{~cm}^{2} \mathrm{~g}^{-1}$. According to Ossenkopf \& Henning (1994), this should be appropriate for $\nu \sim 350 \mathrm{GHz}$ and dust mixed with gas at density $\sim 10^{5}-10^{6} \mathrm{~cm}^{-3}$, but this value is uncertain by a factor of $\sim 2$.

Finally, we combine the dust surface density with an adopted dust-to-gas mass ratio (DGR) of 1-to-100, approximately the Milky Way value and similar to the value found for starburst galaxies by Wilson et al. (2008). Then our estimate of the central gas surface density for each peak is:

$$
\Sigma_{\mathrm{gas}}=\frac{1}{\mathrm{DGR} \kappa_{350 \mathrm{GHz}}} \tau_{350 \mathrm{GHz}}
$$

We then scale this $\Sigma_{\text {gas }}$ by the physical area of the peak, assuming each source to be a two-dimensional Gaussian with the size quoted in Table 1 . Thus, $M_{\mathrm{gas}}=A \Sigma_{\mathrm{gas}}$.

We report the the results in Table 2. We find median $\log _{10} M_{\text {gas }}\left[M_{\odot}\right] \sim 5.0$ and values in the range $\log _{10} M_{\text {gas }}\left[M_{\odot}\right] \sim$ 3.6-5.7.

As with $M_{\star}, M_{\mathrm{gas}}$ represents a nearly linear transformation of the measured source flux at $350 \mathrm{GHz}$. Because we detect the sources at high $\mathrm{S} / \mathrm{N}$, the statistical uncertainties are quite low. Systematic uncertainties in the adopted temperature, mass absorption coefficient, and dust-to-gas ratio dominate the error budget for $M_{\text {gas }}$.

Based on the excitation requirements of the lines that we see, and on the global SED, $T_{\text {dust }}$ seems unlikely to be lower than $\sim 50-60 \mathrm{~K}$ in these dense, heated regions. Because the clusters are likely to be optically thick near the peak of the IR SEDs, we can ask what temperature, along with our measured sizes, would place all of the luminosity of the burst in our targets. Assuming $L=4 \pi r^{2} \sigma_{\mathrm{SB}} T^{4}$, and half of the bolometric IR luminosity from Sanders et al. (2003) to be in the burst, we find that $T_{\text {dust }}$ must be $<160 \mathrm{~K}$. We consider a reasonable plausible range to be $T_{\text {dust }} \sim 60-160 \mathrm{~K}$; given that the ammonia temperatures for the hot components lie in the intermediate part of this range, $T_{\text {dust }} \sim 130 \mathrm{~K}$ with $50 \%$ uncertainty seems like a reasonable assessment.
As noted, $\kappa$ appears uncertain by a factor of $\sim 2$. Allowing a $\sim 30 \%$ uncertainty in the dust-to-gas ratio, the overall uncertainty on the gas mass is likely $\sim 0.4-0.5 \mathrm{dex}$.

For comparison, our assumptions yield an $\approx 30$ times lower gas mass than what one would calculate from the $350 \mathrm{GHz}$ light-to-gas-mass conversion of Scoville et al. (2016). That is, we take the dust in these proto-SSCs to be more emissive and much hotter than typical dust in galaxies. Bearing this in mind, we consider that our gas masses are most likely to be underestimates.

\subsubsection{Dynamical Masses}

We estimate dynamical mass of each source via

$$
M_{\mathrm{VT}}=892 \ell_{\mathrm{FWHM}} \sigma_{v}^{2} .
$$

Here, $\sigma_{v}$ the measured velocity dispersion (in $\mathrm{km} \mathrm{s}^{-1}$ ), $\ell_{\mathrm{FWHM}}$ is the FWHM deconvolved size of the source (both from Table 1), and $M_{\mathrm{VT}}$ is the virial theorem-based dynamical mass in units of solar masses. The prefactor here assumes a density profile $\rho \propto r^{-2}$.

According to this calculation, we find a median dynamical mass of $\log _{10} M_{\mathrm{VT}}\left[M_{\odot}\right] \sim 5.7$ and values in the range $\log _{10} M_{\mathrm{VT}}\left[M_{\odot}\right] \sim 5.1-6.4$.

This calculation assumes that the line widths are due to selfgravity. It corresponds to an upper limit if the velocity dispersion includes some contribution from inflow, outflow, or material unassociated with the source. If our sources break into multiple components at higher resolution, as Sgr B2 does in the Milky Way, then we also expect $M_{\mathrm{VT}}$ to represent an overestimate. We estimate the systematic uncertainty due to unresolved substructure to be $\lesssim 0.3 \mathrm{dex}$, with the sense that our virial masses will be overestimated because we somewhat overestimate the size and line width of the source.

\subsubsection{Comparison of Mass Estimates}

Figure 5 compares our mass estimates. Our sources show gas and stellar masses $\sim 10^{4}$ up to $\sim 10^{6} M_{\odot}$. Just as the sizes that we measure are typical of young cluster sizes (e.g., Ryon et al. 2017), these masses resemble those seen for massive clusters in nearby starbursts (e.g., Whitmore 2003; McCrady et al. 2005). Our sources thus do meet the definition of young massive clusters suggested by, among others, Portegies Zwart et al. (2010) and Longmore et al. (2014).

Our observations suggest a range of gas richness for the targets, but the left panel of Figure 5 shows that gas often contributes a large fraction of the mass. In all but four sources, gas contributes $\gtrsim 50 \%$ of the mass (and bear in mind that we are suspicious of the $M_{\star}$ for sources \#11 and \#12). The median gas mass fraction $\left(M_{\mathrm{gas}} /\left(M_{\mathrm{gas}}+M_{\star}\right)\right.$ across the sample is $\sim 50 \%$, though with significant uncertainties.

The right panel of Figure 5 shows that our virial mass estimates tend to exceed our combined star plus gas estimates by a factor of $\sim 2.5$. Given the uncertainties in the gas and stellar mass estimation, this still represents reasonable agreement. The dynamical mass estimate reinforces that these structures mostly contain $\sim 10^{5}-10^{6} M_{\odot}$ in a region a few parsecs across, with large contributions from both stars and gas. The discrepancy between the two total mass estimates could, in principle, reflect out-of-equilibrium motions (outflows or inflows). Alternatively, it might indicate that the sources are in a nonvirialized dynamical state; for example, the line widths 

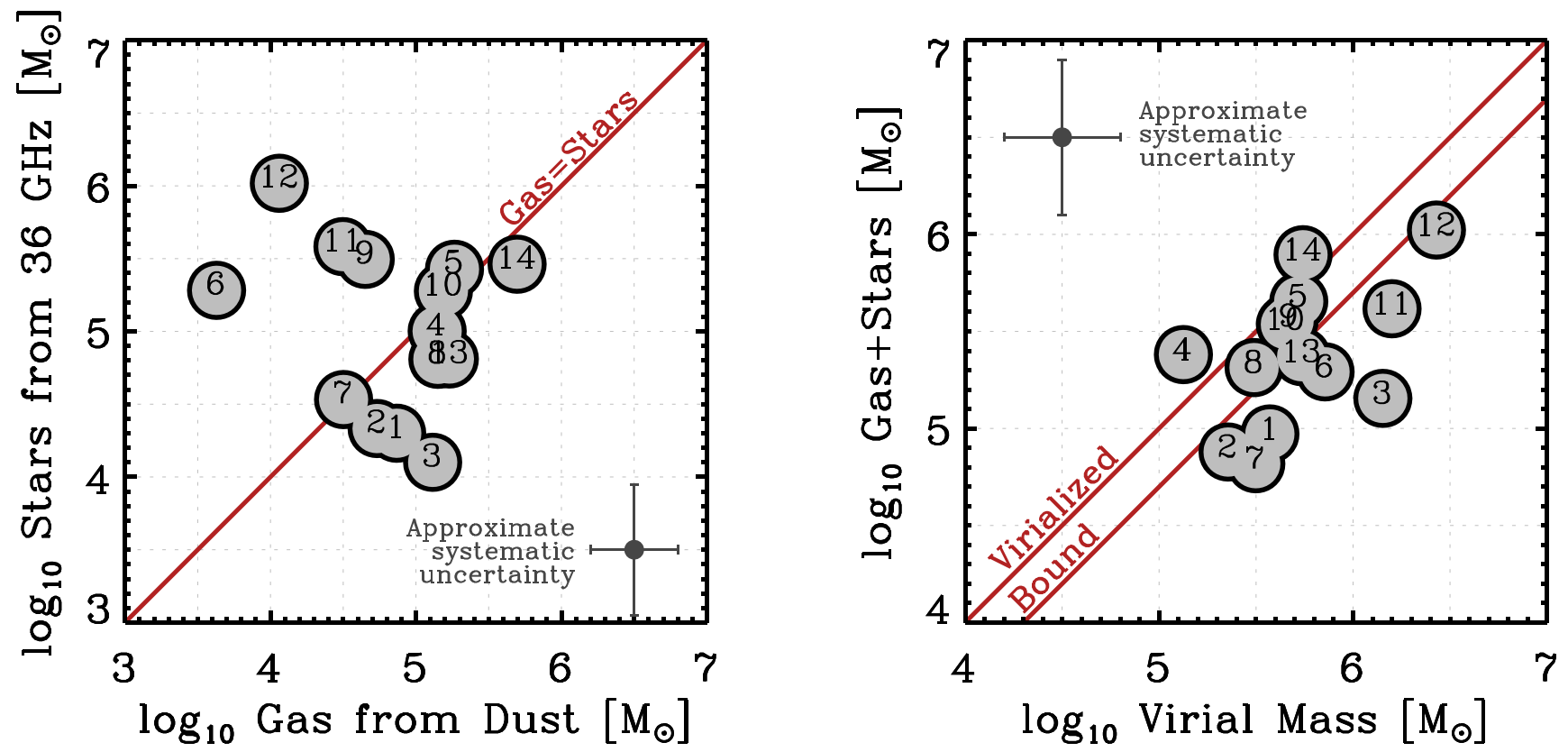

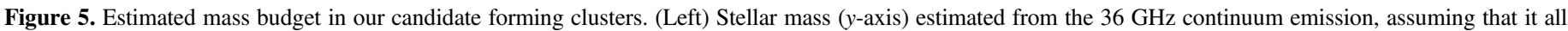

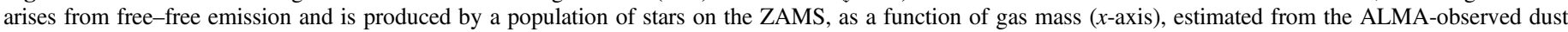

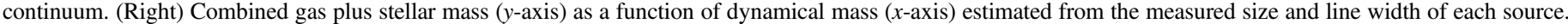

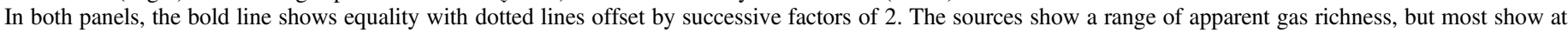

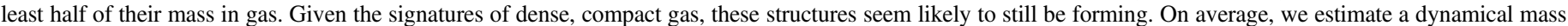

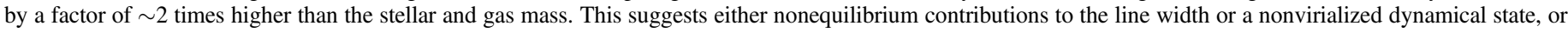
that some of our assumptions underestimate the true mass of gas and stars present.

might reflect blended motion of unassociated sources. Just as likely, the discrepancy between the virial and gas plus stellar masses between the large uncertainties in our mass estimates, especially ionizing photons absorbed by dust and our uncertainties in $\kappa$ and $T_{\text {dust }}$.

\subsubsection{Density and Freefall Time}

Table 2 also reports the total (gas plus stellar) surface density, volume density, and implied gravitational freefall time. These are calculated within the FWHM, so that in two dimensions we divide the mass by 2 and divide by the area at FWHM. In three dimensions, we divide the mass by 3.4 before dividing by the volume at the FWHM.

The median surface density is $\log _{10} \Sigma_{\text {gas }+\star}\left[M_{\odot} \mathrm{pc}^{-2}\right] \sim 4.5$ and values lie in the range of 3.7-5.1. Recasting these values in terms of mass surface density from the edge to the center of the structure (i.e., converting units and dividing by 2), our calculations imply a median of $\sim 3.4 \mathrm{~g} \mathrm{~cm}^{-2}$ from the center to the cluster edge and a range $0.5-14.0 \mathrm{~g} \mathrm{~cm}^{-2}$. These values resemble those found in the Milky Way for other regions of high-mass star formation (e.g., see McKee \& Ostriker 2007). The high end of our range of measured surface densities approaches the $\sim 20 \mathrm{~g} \mathrm{~cm}^{-2}$ maximum surface density (now through the whole object, not center to edge) for stellar systems found by Hopkins et al. (2010). On average, these protoclusters are a factor of $\sim 3$ of below this maximum surface density.

The median gas plus stellar volume density in our targets is $\log _{10} \rho_{\text {gas }+\star}\left[M_{\odot} \mathrm{pc}^{-3}\right] \sim 4.0 \quad$ (range $3.4-4.5$ ) in units of $M_{\odot} \mathrm{pc}^{-3}$. This would correspond to a median $n_{H} \sim 10^{5} \mathrm{~cm}^{-3}$ if all of the material were in molecular gas. The gravitational freefall times implied by these densities will be $\log _{10} \tau_{\mathrm{ff}} \sim 4.9$ (range 4.5-5.3) yr.
Considering only the gas mass, the implied surface densities for our sources would correspond to a median $~ 500 \mathrm{mag}$ (range 20-4200 mag) of $V$-band extinction for a Milky Way dust-to-gas ratio (Bohlin et al. 1978). This helps explain why most of our targets do not appear as distinct sources in the HST imaging.

The final column of Table 2 quotes the escape velocity $\sqrt{2 G M / r}$ calculated within the three-dimensional FWHM of the source using the gas plus stellar masses. Using the data reported in the tables:

$$
v_{\mathrm{esc}}=\sqrt{\frac{2 G\left(\frac{M_{\star}+M_{\mathrm{gas}}}{3.4}\right)}{\left(\frac{\ell_{\mathrm{FWHM}}}{2}\right)}}
$$

where factors of 3.4 account for the fraction of mass inside the FWHM of a three-dimensional Gaussian and a factor of 2 converts from FWHM to HWHM. Again $\ell_{\text {FWHM }}$ refers to the FWHM, deconvolved size of the source from Table 1.

These $v_{\mathrm{esc}}$ for all of our sources exceeds the $\sim 10 \mathrm{~km} \mathrm{~s}^{-1}$ sound speed expected for photoionized gas. As a result, the clusters should match the definition for young massive protoclusters from Bressert et al. (2012).

\subsection{Notes on Individual Sources}

As already mentioned, sources \#11 and \#12 sit in a complex region. Much of the $36 \mathrm{GHz}$ flux that we measure may not directly associated with these clusters. We will treat these two sources with caution when drawing conclusions about the population as a whole. As also mentioned, source \#6 has a high $36-350 \mathrm{GHz}$ ratio. This could imply a more evolved cluster. However, source \#6 is also our faintest source and the formal uncertainty in $f_{\mathrm{ff}}$ is quite high (see Table 1). Moreover, 
the contrast with local background or extended features associated with the bright, nearby source \#5 is poorer than for our other targets.

Several sources show complex line profiles. Sources \#3 and \#4 represent the clearest examples, but sources \#2 and \#13 also show significant non-Gaussian structure. We might expect both inflow and outflow during the evolution of a protocluster, leading to P-Cygni or reverse P-Cygni profiles. Peak \#4 shows some indication of this. We might also expect some of our sources to break up into collections of smaller objects, as would be the case for Sgr B2. This might help explain the profiles in peaks \#2 and \#3. Some of the central dips seen in the profiles (e.g., \#3 and \#13) could alternatively reflect absorption from colder, denser gas.

Based on a first look at even higher resolution ALMA imaging obtained during review of the article, our sources appear to represent the main bright point sources even at $<1 \mathrm{pc}$ resolution. Many sources are associated with smaller satellite sources, and the region around sources \#10, \#11, and \#12 contains at least six discrete point sources, with our three candidate protoclusters the brightest. These data will be presented, when the science is ready, in a future article.

\section{Discussion}

We identify 14 candidate young SSCs in the inner region of NGC 253. How much of the star formation in the burst can these sources account for? What can we say about the efficiencies and timescales associated with these sources? Given such intense concentrations of gas and young stars, what can we infer about feedback in these sources?

\subsection{Timescales and Relation to the Starburst}

The central burst in NGC 253 forms $\sim 2 M_{\odot} \mathrm{yr}^{-1}$ (Bendo et al. 2015; Leroy et al. 2015). How much of that can be attributed to these sources?

Fraction of ionizing photons and IR luminosity from these sources. We estimate a total ionizing photon production of $Q_{0} \sim 1.2 \times 10^{53} \mathrm{~s}^{-1}$ from our targets, with half of this coming from sources $\# 11$ and $\# 12$. Bendo et al. (2015) find $Q_{0}=3.2 \pm 0.2 \times 10^{53} \mathrm{~s}^{-1}$ for the whole burst. Thus, our sources may produce between $20 \%$ and $40 \%$ of the total ionizing photons in the burst. Accounting for absorption of ionizing photons by dust, which must be more significant in our targets than any less embedded population, would increase this fraction.

An analogous case holds for the bolometric luminosity. If $50 \%$ of the total IR luminosity measured by Sanders et al. (2003) arises from the nuclear region, then $L_{\mathrm{TIR}} \sim 1.8 \times 10^{10} L_{\odot}$ for this region. We estimate the contribution of our sources to this value by taking the light-to-mass ratio of a ZAMS population to be $\Psi \equiv L_{\star} / M_{\star} \sim 1000 L_{\odot} M_{\odot}^{-1}$ and adopting the $M_{\star}$ calculated from the $36 \mathrm{GHz}$ emission. In this case, our clusters together contribute $\sim 17 \%$ of the bolometric luminosity of the burst; if we neglect sources \#11 and \#12, this decreases to $\sim 9 \%$.

Relevant timescales. Several distinct timescales should combine to produce our observations. First, the timescale for cluster formation is the time spent in this compact, gas-rich phase. Skinner \& Ostriker (2015) find a typical timescale of $\sim 5 \tau_{\text {ff }}$ for cluster formation. The forming clusters simulated by Skinner \& Ostriker (2015) do evolve over this time. The phase in which gas is actively collapsing to make stars is even shorter, $1-2 \tau_{\text {ff }}$, and the surrounding gas is dispersed by $\sim 8 \tau_{\text {ff. }}$ They measure their $\tau_{\text {ff }}$ averaged over their $r=10 \mathrm{pc}$ cloud, with $\tau_{\text {ff }} \sim 0.5$ Myr. We consider smaller scales and find freefall times of $\sim 10^{5} \mathrm{yr}$ (Table 2). Assuming the Skinner \& Ostriker (2015) results to scale with the freefall time, this implies a visibility timescale for forming clusters of $\sim 8 \times 10^{5} \mathrm{yr}$.

Second, ionizing photon production declines rapidly after $\sim 3-5 \times 10^{6} \mathrm{yr}$. This should be the timescale to produce the overall $Q_{0}$ in the burst. Third, IR or bolometric luminosity is produced over a longer timescale than ionizing photons, with a single stellar population still producing significant light for many tens of megayears.

This short cluster formation timescale, $\sim 10^{6} \mathrm{yr}$, implies that a large amount fraction of stars in the burst are born in clusters (see next section). Our observations do support the idea that the clusters are young. Below, we show that the total radial momentum in these clusters appears low relative to their stellar mass. This implies that feedback has not yet unbound the protoclusters, consistent with an age young enough that a large amount of SNe have not yet gone off. The clusters also show signs of ionizing photon production from compact regions. Still, these radial momentum limits and the presence of ionizing photons only place hard limits of $\lesssim 5-10 \mathrm{Myr}$ on the age of the clusters. The value of $\sim 8 \tau_{\text {ff }} \sim 1$ Myr should be viewed as a key assumption motivated by theory (Skinner \& Ostriker 2015).

Are most stars in the burst born in clusters? The estimated timescale for cluster formation, $\sim 10^{6} \mathrm{yr}$, is $\sim 20 \%-30 \%$ of the timescale for ionizing photon production. If all stars are born in clusters, we expect $20 \%-30 \%$ of the ionizing photons coming from the burst at any given time to arise from still-forming clusters. In this case, our observations agree with a large fraction $(\sim 100 \%)$ of stars being born in the burst proceeding through a phase like what we see.

Equivalently, we can see that the clusters might supply order unity of the star formation simply from their masses. Neglecting sources \#11 and \#12, which have questionable ionization and/or emission mechanisms, we find a total mass within the clusters of $M_{\text {gas }+\star} \approx 3 \times 10^{6} M_{\odot}$, split approximately equally between gas and stars. Combining this total mass with the $8 \tau_{\mathrm{ff}} \sim 10^{6} \mathrm{Myr}$ cluster formation timescale based on Skinner \& Ostriker (2015), then our observed sources can already account for more than the total $\sim 2 M_{\odot} \mathrm{yr}^{-1}$ star formation rate in the burst. This assumes continuous star formation at the time-average rate, leverages our assumed cluster formation timescale, adopts a $100 \%$ gas to star conversion efficiency, and relies on our somewhat uncertain mass estimates. All of these assumptions likely break down in detail, but the calculation shows that the clusters represent a large fraction of the mass that the burst has likely formed over the last $\sim 10^{6} \mathrm{yr}$.

Observations and theory both predict a larger fraction of star forms born in clusters in regions of high star formation surface density (e.g., Kruijssen 2012; Johnson et al. 2016; Ginsburg \& Kruijssen 2018). The nucleus of NGC 253 has one of highest star formation surface densities in the local universe. Finding $\sim 100 \%$ of the stars to be born in clusters in this extreme environment thus qualitatively matches expectations.

A plausible scenario. Our observations appear to be consistent with a scenario in which most of the stars in the burst form in massive young clusters. The formation process 
last for $\sim 1 \mathrm{Myr}$, after which feedback disperses the immediate natal cloud (Skinner \& Ostriker 2015). After this, the clusters remain present, but without an associated large gas reservoir. They will still be invisible in the near-IR, hiding behind the high overall extinction in the region. They will also continue to produce ionizing photons, contributing to the overall $Q_{0}$ in the burst inferred from free-free and radio recombination line emission (Bendo et al. 2015). Meanwhile, their corresponding $\mathrm{H}$ II regions would grow in size, fading in surface brightness and becoming much more difficult to pick up in our interferometric radio continuum maps. Eventually, they might evolve in analogs to the older, visible clusters seen at larger radii by Fernández-Ontiveros et al. (2009).

We expect the strong feedback that drives the X-ray and molecular gas winds (Strickland et al. 2002; Bolatto et al. 2013; Walter et al. 2017) to occur after the embedded young cluster phase that we observe. After $\sim 10 \mathrm{Myr}$, many massive stars will explode as SNe. These explosions may trigger both the hot and cold outflows. A scenario in which strong feedback occurs well after the embedded phase agrees with our observations, which show that the protocluster candidates are approximately gravitationally bound at $2 \mathrm{pc}$ scales, with no evidence for high-velocity line wings in their spectra. In this case, the clusters that we observe now are not the immediate drivers of the outflows observed in Bolatto et al. (2013), Walter et al. (2017), and Zschaechner et al. (2018). They may, however, drive similar outflows in the future.

Lower mass clusters. We observe candidate protoclusters down to a combined gas plus stellar mass of $\log _{10} M_{\text {gas }+\star} \sim 4.8$. The cluster mass function is often taken to have equal power per decade (e.g., Portegies Zwart et al. 2010). Taking the cutoff for young massive clusters as $\sim 10^{4} M_{\odot}$ (Portegies Zwart et al. 2010; Bressert et al. 2012; Longmore et al. 2014), there may be as much mass in low-mass unidentified clusters as in the sources that we study. It is likely that many of these lower mass sources will be substructures still unresolved by our $1.9 \mathrm{pc}$ beam, analogous, for example, to Sgr B2. In this case, they would already be counted in our bookkeeping. However, we also likely miss some peaks that remain blended at our resolution or show too weak a contrast against the background to be detected by our peak-finding algorithm. As a result, there may be even more cluster formation in our region of interest than we observe here. This point should be addressed by ongoing higher resolution ALMA observations.

Of course, if twice as much mass - and ionizing photons and bolometric luminosity-are present in clusters outside our sources, then our bookkeeping above breaks. This could indicate a longer timescale for cluster formation or it might reflect that we have systematically overestimated the mass of the clusters.

Lower limit. As emphasized, the cluster formation timescale remains uncertain. Our mass estimates are also uncertain at the factor of 2 level. Given that all of the sources show $36 \mathrm{GHz}$ flux and that at least $20 \%$ of the ionizing photon production occurs in our sources, a reasonable limiting case is that the visibility lifetime for the clusters equals the $\sim 3-5 \mathrm{Myr}$ ionizing photon production time and that $\sim 20 \%$ of the stars in the burst form in these structures. Even in this limiting case, the burst represents a prodigious cluster production factory, far more extreme than what we see around us in the Milky Way.

\subsection{Likely High Efficiency per Freefall Time}

We find $M_{\star} \sim M_{\text {gas }}$ and freefall times $t_{\mathrm{ff}} \sim 10^{5} \mathrm{yr}$ based on the combined gas plus stellar mass in the clusters. Assuming that no mass has escaped from the cluster since its initial formation, then $M_{\star} \sim M_{\text {gas }}$ implies an overall efficiency of $\sim 50 \%$. That is, $50 \%$ of the initial total mass is now in stars. Following the argument above that cluster formation occurs over $\sim 5 t_{\mathrm{ff}}$, this implies an efficiency per freefall time of $>10 \%$, again assuming no mass loss. If we adopt a visible lifetime of $\sim 1 \mathrm{Myr}$ according to the fraction of ionizing photons seen in the sources, this would instead imply $\sim 5 \%$ of the gas mass converted to stars per freefall time.

Note that some mass loss may already have occurred (e.g., as might be expected following Thompson \& Krumholz 2016), in which case the efficiency per freefall time would be lower than we calculate. To have a $\sim 1 \%$ efficiency per freefall time, as is observed at larger scales (e.g., Krumholz \& Tan 2007; Krumholz et al. 2012; Utomo et al. 2018), several times the currently observed gas must have already been expelled; however, this seems unlikely based on the observed line widths.

\subsection{High Infrared Opacity}

The deconvolved $350 \mathrm{GHz}$ peak brightness temperatures associated with our sources are high, often $\sim 10 \mathrm{~K}$ and in a few cases $20-40 \mathrm{~K}$ or more. We do not know the true dust temperature, but our arguments above suggest that it cannot be much more than $\sim 130 \mathrm{~K}$ on average. In this case, the dust optical depth at $350 \mathrm{GHz}$ is already $\tau \sim 0.1$ in many of our compact sources.

Opaque at infrared wavelengths. For dust, $\tau \propto \nu^{\beta}$ with $\beta \sim 1.5-2$ in the far-IR and submillimeter wavelengths. Combined with the significant $\tau$ at $350 \mathrm{GHz}$, this implies that dust continuum emission from our compact sources will be optically thick for wavelengths shorter than $\lambda \sim 200-300 \mu \mathrm{m}$. They will have a factor of $\sim 50-100$ higher optical depth at $\lambda \sim 100 \mu \mathrm{m}$ compared to $350 \mathrm{GHz}(855 \mu \mathrm{m})$. This yields optical depths $\tau \sim 5-10$ at $100 \mu \mathrm{m}$, and much larger near the implied peak of the dust SED at $\sim 20-30 \mu \mathrm{m}$.

With such high optical depths, these cluster-forming structures might be expected to have large IR photospheres. The regions could appear much larger at IR wavelengths than at submillimeter wavelengths, so that resolving them is possible only with ALMA. Clumpy substructure might render this a more local effect, so that the gross morphology of the sources does not change, but the opacity effects must be present at some scale. This clumpy substructure might be expected from comparing the mean particle densities, $n \sim 10^{5} \mathrm{~cm}^{-3}$, with the typical densities needed to excite the bright $\mathrm{H}^{13} \mathrm{CN}(4-3)$ and CS (7-6) emission we measure, which requires densities $n \sim 10^{7} \mathrm{~cm}^{-3}$.

Significant infrared radiation pressure force. This high opacity at IR wavelengths also implies a strong radiation pressure force exerted by the cluster stars on the surrounding gas (Murray et al. 2010). For spherical systems optically thick to stellar radiation, the stellar radiation creates an outward force $L_{\star} / c$, with $L_{\star}$ the bolometric luminosity. For systems that are optically thick in the IR, the reprocessed IR light also contributes to this outward force. This force due to IR radiation force exceeds the force associated with the primary stellar radiation by a factor equal to the Rosseland mean optical depth 
$\sim \tau_{\text {IR }}$. The high $\tau_{\text {IR }}$ in our clusters thus implies a strong radiation pressure force on the surrounding gas.

Effect of radiation pressure on cluster formation. How does this high radiation pressure affect cluster formation? Following Murray et al. (2010) and Skinner \& Ostriker (2015), for a spherical system centered on a star cluster, the ratio of the IR radiation force to the gravitational force from the stars is

$$
f_{\mathrm{Edd}, \mathrm{IR}}=\frac{\kappa_{\mathrm{IR}, \mathrm{gas}} F_{\mathrm{IR}} / c}{G M_{*} / r^{2}}=\frac{\kappa_{\mathrm{IR}, \mathrm{gas}} \Psi}{4 \pi c G},
$$

where $F_{\mathrm{IR}}=L_{\star} /\left(4 \pi r^{2}\right)$ is the IR flux, assuming all starlight to be reprocessed into the IR. Here $\kappa_{\mathrm{IR} \text {,gas }}$ is the mass absorption coefficient per unit gas mass; note the difference from above where we discuss the mass absorption coefficient of dust alone. Thus, $\kappa_{\mathrm{IR} \text {,gas }}$ includes both the dust properties and the dust-togas ratio. Here $\Psi \equiv L_{\star} / M_{\star}$ refers to the light-to-mass ratio of the central stellar population.

If $f_{\text {Edd,IR }}>1$, then the radiation pressure force exceeds gravity and we might expect the collapse to halt. Skinner \& Ostriker (2015) demonstrated this using numerical radiation hydrodynamic simulations, showing that when $f_{\text {Edd,IR }}>1$, the SFE is limited to $\sim 50 \%$. Their simulations also showed that the radiation field cannot limit collapse in turbulent, SSC-forming clouds when $f_{\text {Edd,IR }}<1$ (see also Tsang \& Milosavljevic 2017).

The stellar population sets $\Psi$, whereas $\kappa_{\mathrm{IR} \text {,gas }}$ is set by dust properties and the dust abundance relative to gas. Adding gas to the system increases the total dust opacity, leading to a higher $\tau_{\text {IR }}$ and more support from radiation pressure. At the same time, however, adding gas to the system increases the weight of gas. Because these two effects balance, a high $\tau_{\text {IR }}$ does not necessarily imply anything about force balance in the cluster (though there can be an indirect dependence of $\kappa_{\mathrm{IR} \text {,gas }}$ on $\tau_{\text {IR }}$ through the dust temperature; Krumholz \& Thompson 2012).

For a ZAMS with a Kroupa IMF, $\Psi \sim 2000 \mathrm{erg} \mathrm{s}^{-1} \mathrm{~g}^{-1}$ $\sim 1000 L_{\odot} M_{\odot}^{-1}$. For temperature range and dust abundance relevant to our clusters, Semenov et al. (2003) find a Rosseland mean opacity $\kappa_{\mathrm{IR} \text {,gas }} \lesssim 5 \mathrm{~cm}^{2} \mathrm{~g}^{-1}$. In this case, $f_{\text {Edd }} \lesssim 0.4$ and stellar gravity would exceed the IR radiation pressure force by a factor $\gtrsim 2$. In this case, radiation pressure would help support the cloud against collapse but not supply all of the support or tear the cloud apart. Including gas self-gravity would only strengthen the effects of gravity relative to radiation pressure.

This situation could change if $\Psi>1000 L_{\odot} M_{\odot}^{-1}$ (e.g., due to a top-heavy IMF). Top-heavy IMFs have been claimed in 30 Doradus (Schneider et al. 2018) and the proto-SSC in NGC 5253 (Turner et al. 2017). Alternatively, if the gas associated with the clusters has a higher than Galactic dust-togas ratio, or unusually opaque grains, $\kappa_{\mathrm{IR}}$ would be higher than assumed above.

We find virial masses within a factor of $\sim 2$ of $M_{\star}+M_{\text {gas }}$. This supports a scenario in which the clusters are gravitationally bound in approximate equilibrium. It appears that radiation forces, though certainly enhanced by a high $\tau_{\mathrm{IR}}$, at most balance gravity at the present time, consistent with the expectations above. At present, we lack independent constraints on the dust-to-gas ratio, nor do we independently measure $L_{\star}$ and $M_{\star}$.

\subsection{Limits on Feedback from Observed Radial Momentum}

The correspondence between virial masses and $M_{\star}+M_{\text {gas }}$ implies that gravity approximately balances the outward force in our clouds. Given enough time, both $\mathrm{SNe}$ and stellar winds can inject enough momentum to unbind the gas and drive a radial expansion. The contrast between the observed radial motions in our sources and the expected momentum injection from $\mathrm{SNe}$ and stellar winds provides additional indirect support for the idea that our sources are young.

Observed limits on radial momentum. Taking all motions to be radial and outward, the momentum per unit stellar mass for an expanding spherical system is

$$
\frac{p_{r}}{M_{\star}} \equiv \sqrt{3} \sigma_{v} \frac{M_{\mathrm{gas}}}{M_{\star}} .
$$

This $p_{r}$ will be the maximum radial momentum compatible with an observed velocity dispersion $\sigma_{v}$ and gas mass $M_{\text {gas }}$. Normalizing by $M_{\star}$ allows a direct comparison with input from SNe and stellar winds, which both scale with stellar mass.

We report $p_{r} / M_{\star}$ limits for our sources in Table 2 . We find mostly $p_{r} / M_{\star}<100 \mathrm{~km} \mathrm{~s}^{-1}$, with the largest value $\sim 400 \mathrm{~km} \mathrm{~s}^{-1}$ for source $\# 3$, which has a high gas mass relative to its stellar mass and also a line profile suggestive of significant substructure (Figure 3).

Momentum from supernova feedback. Numerical simulations considering clustered $\mathrm{SNe}$ exploding in an inhomogeneous medium find a momentum injection per SN (after cooling and shell formation) of $\sim 10^{5} M_{\odot} \mathrm{km} \mathrm{s}^{-1}$ (e.g., Kim et al. 2017, and references therein). For a Kroupa IMF, with roughly one SN per $100 M_{\odot}$ formed, we expect $p_{\star} / M_{\star} \sim 10^{3} \mathrm{~km} \mathrm{~s}^{-1}$ at late times ( $10 \mathrm{Myr})$. This is an order of magnitude higher than what we observe for most sources, suggesting that SNe have not yet had a significant effect on internal motions.

The large values of $p_{\star} / M_{\star}$ are associated with a long timescale, $t_{\mathrm{SN}} \gtrsim 10 \mathrm{Myr}$. Spreading $p_{\star} / M_{\star} \sim 10^{3} \mathrm{~km} \mathrm{~s}^{-1}$ across this $t_{\mathrm{SN}}$, the mean momentum injection rate, $\left(p_{\star} / M_{\star}\right) / t_{\mathrm{SN}}$, may not exceed the gravitational force, $G M_{\text {gas }+\star} M_{\text {gas }} /\left(M_{\star} r^{2}\right) \sim\left(p_{r} / M_{\star}\right) / t_{\mathrm{ff}}$, especially at early times.

This argument does not preclude any $\mathrm{SNe}$ having gone off. Kornei \& McCrady (2009) note the presence of iron lines in their SSC (our source \#5). Our limits should be read as indicating that the observed momentum in the cluster does not reflect a set of clustered-in space and time- $\mathrm{SNe}$ explosions with sufficient intensity to unbind the clusters. This comparison then places a relatively weak constraint on the object age to be $\lesssim 10$ Myr.

Momentum from stellar winds. For stellar wind feedback, the pressure-driven bubble solution of Weaver et al. (1977) yields a ratio of shell momentum to central cluster mass of

$$
\frac{p_{\star}}{M_{\star}}=65 \mathrm{~km} \mathrm{~s}^{-1} \dot{E}_{w, 34}^{4 / 5} n_{5}^{1 / 5} M_{\star, 5}^{-1 / 5} t_{5}^{7 / 5}
$$

Here, $\dot{E}_{w, 34}$ is the average wind luminosity injected per $M_{\odot}$ of stars in units $10^{34} \mathrm{erg} \mathrm{s}^{-1} ; n_{5}$ is the mean hydrogen density in units $10^{5} \mathrm{~cm}^{-3} ; M_{\star, 5}$ is the cluster mass in units $10^{5} M_{\odot}$; and $t_{5}$ is the cluster age in units $10^{5} \mathrm{yr}$. From Starburst99, $\dot{E}_{w, 34}=1$ (Leitherer et al. 1999). 
This calculation also predicts the radius of the wind-driven bubble. If there are no energy losses and gravity is negligible,

$$
r_{b}=3 \mathrm{pc} \dot{E}_{w, 34}^{1 / 5} n_{5}^{-1 / 5} M_{\star, 5}^{1 / 5} t_{5}^{3 / 5} .
$$

Though we are not yet in a position to measure the relative structure of the ionized gas, molecular gas, and dust, we do not expect $r_{b}$ to exceed our observed source size. In that case, the stellar winds would have cleared out the cold gas.

If the sources in NGC 253 are young $\left(t_{5}<1\right)$, both the momentum injection and bubble size would remain below the observed limits for most sources even without losses. However, if the sources are closer to $\sim 1 \mathrm{Myr}$ in age, as seems likely given their inferred $t_{\mathrm{ff}}$, then the predicted bubble radius and wind momentum input may significantly exceed our observed limits.

Again, it seems likely that the momentum injection from the wind has been balanced by gravity. Comparing the predicted momentum input rate from a wind-blown bubble, $\left(p_{\star} / M_{\star}\right) / t \propto t^{2 / 5}$ to the gravitational force, $\left(p_{r} / M_{\star}\right) / t_{\mathrm{ff}}$, this calculation suggests that the force from winds should exceed that of gravity for $t \sim 10^{6} \mathrm{yr}$. Again, the lack of strong signatures of gas expulsion argue that our sources are young, $\lesssim 10^{6} \mathrm{yr}$. In this case, the effective wind luminosity must be reduced below the expected input value, either by mixing and cooling or by other processes. This is reasonable based on the low X-ray emission observed in somewhat more evolved systems, where the energy in hot gas seems to be far below the value nominally expected from winds, consistent with a reduction in $\dot{E}_{w, 34}$ well below unity (e.g., Harper-Clark \& Murray 2009; Lopez et al. 2011; Rosen et al. 2014).

\section{Summary}

We present new, $\sim 2$ pc resolution ALMA observations of the $350 \mathrm{GHz}$ dust continuum emission from the innermost region of NGC 253, the nearest nuclear starburst. This imaging reveals 14 compact, bright sources. The association of these dust emission peaks with VLA $36 \mathrm{GHz}$ continuum suggests that they already host significant populations of massive young stars (many also appear in the $23 \mathrm{GHz}$ images of Ulvestad \& Antonucci 1997). Despite this, the heavy extinction toward the burst renders all but one of them indistinguishable in near-IR emission. The one known source has previously been identified as a young super star cluster by Watson et al. (1996) and Kornei \& McCrady (2009). Studying these sources, we find the following:

1. Massive, compact sources. We measure source sizes from the ALMA $350 \mathrm{GHz}$ continuum (Table 1). We estimate gas masses based on dust emission, and calculate stellar masses assuming that the observed $36 \mathrm{GHz}$ continuum is free-free emission from a ZAMS population (Table 2).

We find sizes of a few parsecs (FWHM) and estimate total masses $M_{\text {gas }}+M_{\star} \gtrsim 10^{5} M_{\odot}$. We also estimate dynamical masses from the measured sizes and line widths and assuming virialization. The virial masses are typically $\sim 2.5$ times higher than our $M_{\text {gas }}+M_{\star}$ estimates, which are reasonable given the substantial uncertainties on the mass estimation.

2. Likely young super star clusters. These masses and sizes resemble those of young massive clusters seen in the Milky Way and other galaxies (Portegies Zwart et al. 2010; Longmore et al. 2014). In addition, these masses and radii imply escape speeds $>16 \mathrm{~km} \mathrm{~s}^{-1}$. This is larger than the sound speed of photoionized gas, $\sim 10 \mathrm{~km} \mathrm{~s}^{-1}$, so that the sources also match the criteria for young massive protoclusters laid out by Bressert et al. (2012). Clusters in this mass range are often referred to as SSCs.

3. Still in the process of formation. Our estimates of the gas and stellar mass, while uncertain, suggest that gas still contributes a large fraction of the total mass in these objects (Table 1). We observe that the dust emission coincides with $\mathrm{H}^{13} \mathrm{CN}(4-3)$ and $\mathrm{CS}(7-6)$ emission, which are both tracers of dense excited gas. Thus, many of these objects seem likely to still be in the process of formation.

4. Short freefall times and high efficiency. The freefall times implied by the gas plus stellar masses of our sources is short, $\tau_{\text {ff }} \sim 10^{5} \mathrm{yr}$. Given theoretical expectations of $\sim 8 \tau_{\text {ff }}$ for the cluster formation timescale (Skinner \& Ostriker 2015), this implies that the sources are young. Stars typically represent half of the mass in our sources, implying both a high net efficiency and a high efficiency per freefall time. Note that this statement does not take into account possible mass loss. Any mass lost from the system would decrease the both the net star formation efficiency and the efficiency per freefall time.

5. A large fraction of stars form in these sources. At least $20 \%$ of the ionizing photon production in the burst appears associated with these sources. This represents a firm lower limit on the fraction of stars that form in such sources. If the cluster formation timescale is short compared to the time for stars to produce ionizing photons, then an even larger fraction of star formation may proceed through this phase. Accounting for a short cluster formation timescale and the possibility of lower mass, still-unidentified clusters, on the order of $\sim 100 \%$ of the stars in the burst, could be produced in these sources. This number remains uncertain because of uncertainties in both the mass estimates and timescales.

6. Opaque in the infrared. These sources have high brightness temperature. Given plausible dust temperatures, they also have moderate $(\tau \sim 0.1)$ optical depths at submillimeter wavelengths. This implies large optical depths near the peak in the IR near the likely peak of their SEDs. In turn, this implies a significant IR radiation pressure force. Given the reasonable agreement between the dynamical masses and our other estimates of $M_{\text {gas }}+M_{\star}$, this force may help support the clouds but is not unbinding them. This agrees with theoretical expectations.

7. Young based on large gas fraction and being approximately bound. Our sources retain a large fraction of their mass in gas (as evidenced by the dust continuum). They also appear to be approximately gravitationally bound. We also calculate limits on the radial momentum in our sources and compare them to expectations from $\mathrm{SNe}$ and stellar wind feedback. Our sources have lower radial momentum and smaller sizes than expected from either clustered $\mathrm{SNe}$ or stellar winds acting over many megayears, though losses in wind energy may be important. All of these pieces of evidence suggest that the sources are young enough that feedback has not managed to unbind the gas from the cluster. 
Given the brightness of these sources, ALMA and the Jansky VLA both offer the prospect for even more detailed detailed follow-up. Higher resolution dust observations are already underway, as is the construction of full radio-to-millimeter SEDs for each source (building on Ulvestad \& Antonucci 1997; Mohan et al. 2005, and leveraging new ALMA and VLA work).

It will also be important to link these structures larger context of a the burst. In the Milky Way's Central Molecular Zone, star formation has been linked to the orbital paths of individual clouds (e.g., Kruijssen et al. 2014). The linear distribution of the sources we see suggests an underlying barlike structure (see Paglione et al. 2004; Leroy et al. 2015) or loosely wound arms. It may be possible to link this structure to the triggering of star formation. More generally, we do not see clear analogs for these structures in the Milky Way. This might be because NGC 253 sits at a different part of some long-term nuclear fueling cycle (e.g., Krumholz et al. 2017). A more detailed comparison of the two systems (building on Sakamoto et al. 2011) could help reveal the overall triggers and likely duty cycle of the burst. This might also help reveal the fate of the protoclusters after they disappear from our ALMA and VLA imaging, and perhaps link them to clusters seen on larger scales outside the area we study (Fernández-Ontiveros et al. 2009).

We thank the anonymous referee for a constructive report that improved the article. We also thank Gerhardt Meurer and Mark Krumholz for useful feedback during revision.

This article makes use of the following ALMA data: ADS/ JAO.ALMA\#2015.1.00274.S. ALMA is a partnership of ESO (representing its member states), NSF (USA) and NINS (Japan), together with NRC (Canada), NSC and ASIAA (Taiwan), and KASI (Republic of Korea), in cooperation with the Republic of Chile. The Joint ALMA Observatory is operated by ESO, AUI/NRAO and NAOJ. The National Radio Astronomy Observatory is a facility of the National Science Foundation operated under cooperative agreement by Associated Universities, Inc.

The work of A.K.L. is partially supported by the National Science Foundation under grants No. 1615105, 1615109, and 1653300. The work of A.D.B. is supported in part by the NSF under grant No. AST-1412419. The work of E.C.O. is supported by the NSF under grant No. AST-1713949. The work of T.A.T. is supported in part by NSF Award No. 1516967. The $H S T$ data presented in this paper were acquired under program HST-GO-13730 with support provided by NASA through a grant from the STScI. STScI is operated by the Association of Universities for Research in Astronomy, Inc., under NASA contract NASS-26555.

We acknowledge the usage of the Extragalactic Distance Database $^{14}$ (Tully et al. 2009), the HyperLeda database ${ }^{15}$ (Makarov et al. 2014), the NASA/IPAC Extragalactic Database, ${ }^{16}$ and the SAO/NASA Astrophysics Data System. ${ }^{17}$

Facilities: ALMA, VLA, HST.

Software: CASA (McMullin et al. 2007), IDL, CPROPS ${ }^{18}$ (Rosolowsky \& Leroy 2006; Leroy et al. 2015).

\footnotetext{
14 http://edd.ifa.hawaii.edu/index.html

15 http://leda.univ-lyon1.fr

16 http://ned.ipac.caltech.edu

17 http://www.adsabs.harvard.edu

18 https://github.com/akleroy/cpropstoo
}

\section{ORCID iDs}

Adam K. Leroy (10 https://orcid.org/0000-0002-2545-1700 Alberto D. Bolatto (i) https://orcid.org/0000-0002-5480-5686 Eve C. Ostriker (1) https://orcid.org/0000-0002-0509-9113 Fabian Walter (1) https://orcid.org/0000-0003-4793-7880 Adam Ginsburg (i) https://orcid.org/0000-0001-6431-9633 Nico Krieger (1) https://orcid.org/0000-0003-1104-2014 Rebecca C. Levy (1) https://orcid.org/0000-0003-2508-2586 David S. Meier (10) https://orcid.org/0000-0001-9436-9471 Elisabeth Mills (i) https://orcid.org/0000-0001-8782-1992 Jürgen Ott (i) https://orcid.org/0000-0001-8224-1956 Erik Rosolowsky (i) https://orcid.org/0000-0002-5204-2259 Sylvain Veilleux (1) https://orcid.org/0000-0002-3158-6820 Laura K. Zschaechner (10 https://orcid.org/0000-00029919-8672

\section{References}

Ando, R., Nakanishi, K., Kohno, K., et al. 2017, arXiv:1710.01432 Bendo, G. J., Beswick, R. J., D’Cruze, M. J., et al. 2015, MNRAS, 450, L80 Binder, B. A., \& Povich, M. S. 2018, arXiv:1808.00454

Bohlin, R. C., Savage, B. D., \& Drake, J. F. 1978, ApJ, 224, 132

Bolatto, A. D., Warren, S. R., Leroy, A. K., et al. 2013, Natur, 499, 450

Bressert, E., Ginsburg, A., Bally, J., et al. 2012, ApJL, 758, L28

Caplan, J., \& Deharveng, L. 1986, A\&A, 155, 297

Dale, D. A., Cohen, S. A., Johnson, L. C., et al. 2009, ApJ, 703, 517

Draine, B. T. 2011, Physics of the Interstellar and Intergalactic Medium

Eldridge, J. J., Stanway, E. R., Xiao, L., et al. 2017, PASA, 34, e058

Fernández-Ontiveros, J. A., Prieto, M. A., \& Acosta-Pulido, J. A. 2009, MNRAS, 392, L16

Fukui, Y., Torii, K., Ohama, A., et al. 2016, ApJ, 820, 26

Gao, Y., \& Solomon, P. M. 2004, ApJ, 606, 271

Ginsburg, A., Bally, J., Barnes, A., et al. 2018, ApJ, 853, 171

Ginsburg, A., Bressert, E., Bally, J., \& Battersby, C. 2012, ApJL, 758, L29

Ginsburg, A., \& Kruijssen, J. M. D. 2018, ApJL, 864, L17

Gorski, M., Ott, J., Rand, R., et al. 2017, ApJ, 842, 124

Harper-Clark, E., \& Murray, N. 2009, ApJ, 693, 1696

Herrera, C. N., Boulanger, F., Nesvadba, N. P. H., \& Falgarone, E. 2012, A\&A, 538, L9

Holtzman, J. A., Faber, S. M., Shaya, E. J., et al. 1992, AJ, 103, 691

Hopkins, P. F., Murray, N., Quataert, E., \& Thompson, T. A. 2010, MNRAS, 401, L19

Johnson, K. E., Leroy, A. K., Indebetouw, R., et al. 2015, ApJ, 806, 35

Johnson, L. C., Seth, A. C., Dalcanton, J. J., et al. 2016, ApJ, 827, 33

Kim, C.-G., Ostriker, E. C., \& Raileanu, R. 2017, ApJ, 834, 25

Knudsen, K. K., Walter, F., Weiss, A., et al. 2007, ApJ, 666, 156

Kormendy, J., \& Kennicutt, R. C., Jr. 2004, ARA\&A, 42, 603

Kornei, K. A., \& McCrady, N. 2009, ApJ, 697, 1180

Kroupa, P. 2001, MNRAS, 322, 231

Kruijssen, J. M. D. 2012, MNRAS, 426, 3008

Kruijssen, J. M. D., Longmore, S. N., Elmegreen, B. G., et al. 2014, MNRAS, 440, 3370

Krumholz, M. R., Dekel, A., \& McKee, C. F. 2012, ApJ, 745, 69

Krumholz, M. R., Kruijssen, J. M. D., \& Crocker, R. M. 2017, MNRAS, 466,1213

Krumholz, M. R., \& Matzner, C. D. 2009, ApJ, 703, 1352

Krumholz, M. R., \& Tan, J. C. 2007, ApJ, 654, 304

Krumholz, M. R., \& Thompson, T. A. 2012, ApJ, 760, 155

Lee, J. C., Gil de Paz, A., Tremonti, C., et al. 2009, ApJ, 706, 599

Leitherer, C., Schaerer, D., Goldader, J. D., et al. 1999, ApJS, 123, 3

Leroy, A. K., Bolatto, A. D., Ostriker, E. C., et al. 2015, ApJ, 801, 25

Longmore, S. N., Kruijssen, J. M. D., Bastian, N., et al. 2014, Protostars and Planets VI, 291

Longmore, S. N., Walsh, A. J., Purcell, C. R., et al. 2017, MNRAS, 470, 1462

Lopez, L. A., Krumholz, M. R., Bolatto, A. D., Prochaska, J. X., \& Ramirez-Ruiz, E. 2011, ApJ, 731, 91

Makarov, D., Prugniel, P., Terekhova, N., Courtois, H., \& Vauglin, I. 2014, A\&A, 570, A13

Mangum, J. G., Darling, J., Henkel, C., et al. 2013, ApJ, 779, 33

McCrady, N., Graham, J. R., \& Vacca, W. D. 2005, ApJ, 621, 278

McKee, C. F., \& Ostriker, E. C. 2007, ARA\&A, 45, 565 
McMullin, J. P., Waters, B., Schiebel, D., Young, W., \& Golap, K. 2007, in ASP Conf. Ser. 376, Astronomical Data Analysis Software and Systems XVI, ed. R. A. Shaw, F. Hill, \& D. J. Bell (San Francisco, CA: ASP), 127 Meier, D. S., Turner, J. L., \& Schinnerer, E. 2011, AJ, 142, 32

Meier, D. S., Walter, F., Bolatto, A. D., et al. 2015, ApJ, 801, 63

Mohan, N. R., Goss, W. M., \& Anantharamaiah, K. R. 2005, A\&A, 432, 1

Murphy, E. J., Condon, J. J., Schinnerer, E., et al. 2011, ApJ, 737, 67

Murray, N., Quataert, E., \& Thompson, T. A. 2010, ApJ, 709, 191

Ochsendorf, B. B., Zinnecker, H., Nayak, O., et al. 2017, NatAs, 1, 784

Oey, M. S., Herrera, C. N., Silich, S., et al. 2017, ApJL, 849, L1

Ossenkopf, V., \& Henning, T. 1994, A\&A, 291, 943

Paglione, T. A. D., Wall, W. F., Young, J. S., et al. 2001, ApJS, 135, 183

Paglione, T. A. D., Yam, O., Tosaki, T., \& Jackson, J. M. 2004, ApJ, 611, 835

Portegies Zwart, S. F., McMillan, S. L. W., \& Gieles, M. 2010, ARA\&A, 48, 431

Rekola, R., Richer, M. G., McCall, M. L., et al. 2005, MNRAS, 361, 330

Rosen, A. L., Lopez, L. A., Krumholz, M. R., \& Ramirez-Ruiz, E. 2014, MNRAS, 442, 2701

Rosolowsky, E., \& Leroy, A. 2006, PASP, 118, 590

Rybicki, G. B., \& Lightman, A. P. 1986, Radiative Processes in Astrophysics, 400

Ryon, J. E., Gallagher, J. S., Smith, L. J., et al. 2017, ApJ, 841, 92

Sakamoto, K., Mao, R.-Q., Matsushita, S., et al. 2011, ApJ, 735, 19

Sanders, D. B., Mazzarella, J. M., Kim, D., Surace, J. A., \& Soifer, B. T. 2003, AJ, 126, 1607

Schinnerer, E., Böker, T., Emsellem, E., \& Downes, D. 2007, A\&A, 462, L27

Schneider, F. R. N., Sana, H., Evans, C. J., et al. 2018, Sci, 359, 69

Scoville, N., Sheth, K., Aussel, H., et al. 2016, ApJ, 820, 83

Semenov, D., Henning, T., Helling, C., Ilgner, M., \& Sedlmayr, E. 2003, A\&A, 410,611

Shirley, Y. L. 2015, PASP, 127, 299
Skinner, M. A., \& Ostriker, E. C. 2015, ApJ, 809, 187

Sorai, K., Nakai, N., Kuno, N., Nishiyama, K., \& Hasegawa, T. 2000, PASJ, 52,785

Strickland, D. K., Heckman, T. M., Weaver, K. A., Hoopes, C. G., \& Dahlem, M. 2002, ApJ, 568, 689

Tan, Q.-H., Gao, Y., Zhang, Z.-Y., et al. 2018, ApJ, 860, 165

Thompson, T. A., \& Krumholz, M. R. 2016, MNRAS, 455, 334

Tsai, C.-W., Turner, J. L., Beck, S. C., et al. 2006, AJ, 132, 2383

Tsai, C.-W., Turner, J. L., Beck, S. C., Meier, D. S., \& Ho, P. T. P. 2009, AJ, 137,4655

Tsang, B. T.-H., \& Milosavljevic, M. 2017, arXiv:1709.07539

Tully, R. B., Rizzi, L., Shaya, E. J., et al. 2009, AJ, 138, 323

Turner, J. L., \& Beck, S. C. 2004, ApJL, 602, L85

Turner, J. L., Beck, S. C., Crosthwaite, L. P., et al. 2003, Natur, 423, 621

Turner, J. L., Consiglio, S. M., Beck, S. C., et al. 2017, ApJ, 846, 73

Turner, J. L., \& Ho, P. T. P. 1985, ApJL, 299, L77

Ulvestad, J. S., \& Antonucci, R. R. J. 1997, ApJ, 488, 621

Urquhart, J. S., König, C., Giannetti, A., et al. 2018, MNRAS, 473, 1059

Utomo, D., Sun, J., Leroy, A. K., et al. 2018, ApJL, 861, L18

Walter, F., Bolatto, A. D., Leroy, A. K., et al. 2017, ApJ, 835, 265

Watson, A. M., Gallagher, J. S., III, Holtzman, J. A., et al. 1996, AJ, 112, 534

Weaver, R., McCray, R., Castor, J., Shapiro, P., \& Moore, R. 1977, ApJ, 218, 377

Whitmore, B. C. 2003, in A Decade of Hubble Space Telescope Science, Vol. 14 ed. M. Livio, K. Noll, \& M. Stiavelli, 153

Wilson, C. D., Petitpas, G. R., Iono, D., et al. 2008, ApJS, 178, 189

Xiao, L., Stanway, E. R., \& Eldridge, J. J. 2018, MNRAS, 477, 904

Zhang, H.-X., Gao, Y., \& Kong, X. 2010, MNRAS, 401, 1839

Zhang, Z.-Y., Gao, Y., Henkel, C., et al. 2014, ApJL, 784, L31

Zschaechner, L. K., Bolatto, A. D., Walter, F., et al. 2018, arXiv:1809.01160 